\title{
Research Paper \\ Structural Equation Modeling of Intolerance of Uncertainty and Symptoms of Depression and General Anxiety Disorder: Investigation of the Mediating Role of Rumination
}

\author{
${ }^{*}$ Ghasem Abdolpour ${ }^{1}$, Mohammad Nasiri² 0 , Mehrab Mafakheri ${ }^{3}$, Kamyar Mansouri ${ }^{3}$, Reza Abdi ${ }^{4}$
}

1. PhD. in Clinical Psychology, Department of Psychology, Faculty of Humanities, University of Shahed, Tehran, Iran.

2. PhD. in Health Psychology, Department of Clinical Psychology, Faculty of Psychology and Education, University of Kharazmi, Tehran, Iran.

3. MSc. in Psychology, Department of Clinical Psychology, School of Behavioral Sciences and Mental Health (Tehran Institute of Psychiatry), Iran University of Medical Sciences, Tehran, Iran.

4. PhD. in Psychology, Department of Psychology, Faculty of Psychology and Education, Azarbaijan Shahid Madani University, Tabriz, Iran.

\begin{tabular}{|c|c|}
\hline \multirow{5}{*}{$\begin{array}{l}\text { Use your device to scan } \\
\text { and read the article online }\end{array}$} & \multirow[b]{2}{*}{ Citation Abdolpour Gh, Nasiri M, Mafakheri M, Mansouri K, Abdi R. [Structural Equation Modeling of Intolerance of Uncer- } \\
\hline & \\
\hline & tainty and Symptoms of Depression and General Anxiety Disorder: Investigation of the Mediating Role of Rumination (Persian)]. \\
\hline & Iranian Journal of Psychiatry and Clinical Psychology. 2019; 24(4):400-415. http://dx.doi.org/10.32598/ijpcp.24.4.400 \\
\hline & d bi)": http://dx.doi.org/10.32598/ijpcp.24.4.400 \\
\hline
\end{tabular}

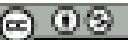

Received: 04 Mar 2018 Accepted: 07 Aug 2018 Available Online: 01 Jan 2019

Keywords: Intolerance of Uncertainty, Rumination Depression, Anxiety disorder, Structural Equation Modeling

\section{A B S TRACT}

Objectives Generalized anxiety disorder and depression, one of the common problems in childhood and adulthood is associated with many problems in the field of health. This study aimed to investigate the mediating role of rumination in the relationship among Intolerance of Uncertainty (IU) and symptoms of depression and General Anxiety Disorder (GAD).

Methods In this descriptive correlational study, a sample of 250 students from Iran University of Medical Sciences were selected using cluster sampling method. The participants responded to intolerance of uncertainty scale and rumination scale, Generalized Anxiety Disorder Questionnaire Inventory-revised (GAD-Q-IV), and Beck depression inventory. The obtained data were analyzed using Structural Equation Modeling (SEM). For statistical analysis, SPSS V. 22 and LISREL 85.9 were used to classify, process, and analyze the data and to investigate the hypotheses of the research.

Results The evaluation of hypothetical model with fit indexes demonstrated that the hypothetical model fits the measurement model $(\mathrm{CFI}=0.98, \mathrm{NFI}=0.96$, and $\mathrm{RMSEA}=0.060$ ).

Structural relations analysis also indicated intolerance of uncertainty indirectly through rumination over depression and directly and indirectly general anxiety disorder.

Conclusion Thus, based on the current research findings, rumination has a mediating role in relationship between intolerance of uncertainty and symptoms of depression and general anxiety disorder. Considering these dimensions, the employed mechanisms can be useful in developing efficacious preventive and therapeutic interventions for depression.

\footnotetext{
Extended Abstract

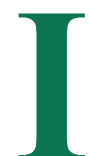

ㄷ...

* Corresponding Author:

Ghasem Abdolpour, PhD.

Address: Department of Psychology, Faculty of Humanities, University of Shahed, Tehran, Iran.

Tel: +98 (938) 7945547

E-mail: g.marand@yahoo.com

including obsessive-compulsive disorder $[9,10]$ and mood disorders [11, 12]. De Jong-Meyer, Beck and Riede [17] in their findings reported that individuals with higher intolerance of uncertainty have greater worry and rumination. In other words, the relationship between Intolerance of Uncertainty (IU), worry and rumination is a causal relationship. Most research in this field has been conducted on patients

\section{tributes to the experience of anxiety in gen-} eral anxiety and ofher anxiety disorders, 
with anxiety (especially general anxiety disorder). For example, studies have indicated that uncertainty is the key in the explanation of concern as the main feature of the general anxiety disorder [27-29]. However, very few studies have examined IU between mood disorders. Because of the similarities and common factors between generalized anxiety disorder and depression, the question is whether the IU is also associated with depression or not. However, this has attracted a relatively less empirical interest in depression.

Spasojevic' and Alloy [36] postulated rumination as a mediator between cognitive risk factors/vulnerabilities and negative psychological outcomes (e.g. depression). Spasojevic' and Alloy indicated that people with cognitive risk factors/ vulnerabilities for depression (e.g. IU, self-criticism) are likely to consistently engage in rumination to cope with their negative mood. Thus, these authors conceptualized rumination as the underlying link (i.e. mediator) between cognitive vulnerability (e.g. IU) and adverse psychological outcomes. Accordingly, the purpose of this study is to present a hypothetical model and test it through Structural Equation Modeling (SEM) approach.

\section{Method}

The present study is a fundamental type and of a descriptive-correlational type in terms of data collection. Considering the minimum sample size required when the variables of the model (in the hypothesized model of the present study, 13 variables) ranges from 10 to 15 , the sample size should be between 200 and 400 [37]. Therefore, the sample size of this study was equal to 250 subjects. To choose the subjects, by cluster sampling method, four colleges (School of Medicine, School of Paramedicine, School of Nursing and Midwifery, School of Public Health) were randomly selected. Then from each college, a number of students were randomly selected to include them in the research.

To test the research hypotheses, the collected data were analyzed using SPSS V. 22 and LISREL 8.85 [42]. The fitness of the hypothesized model was assessed using SEM method. The data analysis was performed using the two-step approach of Anderson and Gerbing [43] as follows: in the first step, Confirmatory Factor Analysis (CFA) was used to assess the fitness of the measurement model, and in the second step, SEM was employed to assess the hypothesized structural model.

\section{Results}

Table 1 presents the matrix of correlation coefficients between the variables of the research. According to the Table 1 , the correlations are significant $(0.549 \geq \mathrm{r} \geq 0.357)$. The measurement model specifies the relationship between the observed and latent variables. The evaluation of the model was done using CFA method. The fit indices of the measurement model (Table 2) presents a satisfactory fitness for this model. Therefore, the observed variables are capable of operating the latent variables.

Table 1. Correlation matrix of research variables

\begin{tabular}{|c|c|c|c|c|}
\hline Variables & 1 & 2 & 3 & 4 \\
\hline Intolerance of uncertainty & 1 & & & \\
\hline Rumination & $0.409 * *$ & 1 & & \\
\hline Depression & $0.357^{* *}$ & $0.549 * *$ & 1 & \\
\hline Anxiety & $0.442 * *$ & $0.462^{* *}$ & $0.427^{* *}$ & 1 \\
\hline
\end{tabular}

Table 2. Fit indices of measurement and structural models

\begin{tabular}{|c|c|c|c|c|c|c|c|c|}
\hline Indice & Chi-square & df & $\chi^{2} / \mathrm{df}$ & RMSEA & SRMR & GFI & CFI & NFI \\
\hline Measurement model & 100.21 & 59 & 1.698 & 0.059 & 0.054 & 0.93 & 0.98 & 0.96 \\
\hline Structural model & 103.59 & 60 & 1.726 & 0.060 & 0.055 & 0.93 & 0.98 & 0.96 \\
\hline
\end{tabular}

Abbreviations: RMSEA; Root Mean Square Error of Approximation; SRMR; Standardized Root Mean square Residual; GFI; Goodness of Fit Index; CFI; Comparative Fit Index; NFI; Normed Fit Index 
Table 3. Bootstrap test results for intermediate relationships

\begin{tabular}{|c|c|c|c|c|c|c|c|}
\hline \multirow[b]{2}{*}{ Independent } & \multicolumn{2}{|l|}{ Variable } & \multicolumn{2}{|c|}{ Bootstrap Bound } & \multirow{2}{*}{ Estimation Error } & \multirow{2}{*}{ Effect Size } & \multirow{2}{*}{$\mathbf{P}$} \\
\hline & Mediating & Dependent & UpperBound & LowerBound & & & \\
\hline $\begin{array}{l}\text { Intolerance of uncer- } \\
\text { tainty }\end{array}$ & Rumination & Depression & 0.469 & 0.205 & 0.069 & 0.337 & 0.05 \\
\hline $\begin{array}{c}\text { Intolerance of uncer- } \\
\text { tainty }\end{array}$ & Rumination & Anxiety & 0.329 & 0.102 & 0.058 & 0.216 & 0.05 \\
\hline
\end{tabular}

In addition, assessing the structural model via the SEM method revealed that all fit indices of the hypothesized model were within the appropriate fitness range. Table 2 presents the fit indices for this model. Figure 1 depicts the hypothetical structural model with its standard coefficients. As seen in Figure 1, intolerance of uncertainty as an exogenous variable affects the rumination with a standard coefficient of 0.50 . In addition, rumination affects general anxiety disorder and depression with standard coefficients of $0.72,0.49$, respectively. In the current study, the Bootstrap test was employed to evaluate the intermediate relationships. In this method, if the upper and lower limits of this test are either positive or negative, and zero is not between these two limits, then the indirect causal path will be significant. Table 3 presents the results of this test.

\section{Discussion}

The purpose of this study was to investigate the mediating role of rumination in the relationship between intolerance of uncertainty with symptoms of depression and general anxi- ety. Structural relations between the variables of the study indicate a good fit of the hypothesized model. To explain these findings, it can be said that those who have a lot of rumination, are susceptible [21] to obscure events, pessimistic forecasts and thoughts [48]. Rumination may maintain or increase the level of uncertainty. In addition, individual mental retardation, unsolved problems, and higher level of uncertainty can persist because rumination disrupts problem solving. Therefore, rumination may increase the link between uncertainty and anxiety intolerance by reinforcing and increasing distress related to uncertainty.

Theoretically, the results of this research provide a supportive framework for Spasovich and Alvoy's conceptual framework of rumination as interface between cognitive vulnerability and negative implications. This effect of rumination as a mediator indicates that people with a high level of uncertainty intolerance tend to chew on disturbances and disorders that worsen symptoms of anxiety and depression. In this study, uncertainty intolerance may lead to negative consequences (such as depression, anxiety) through passive

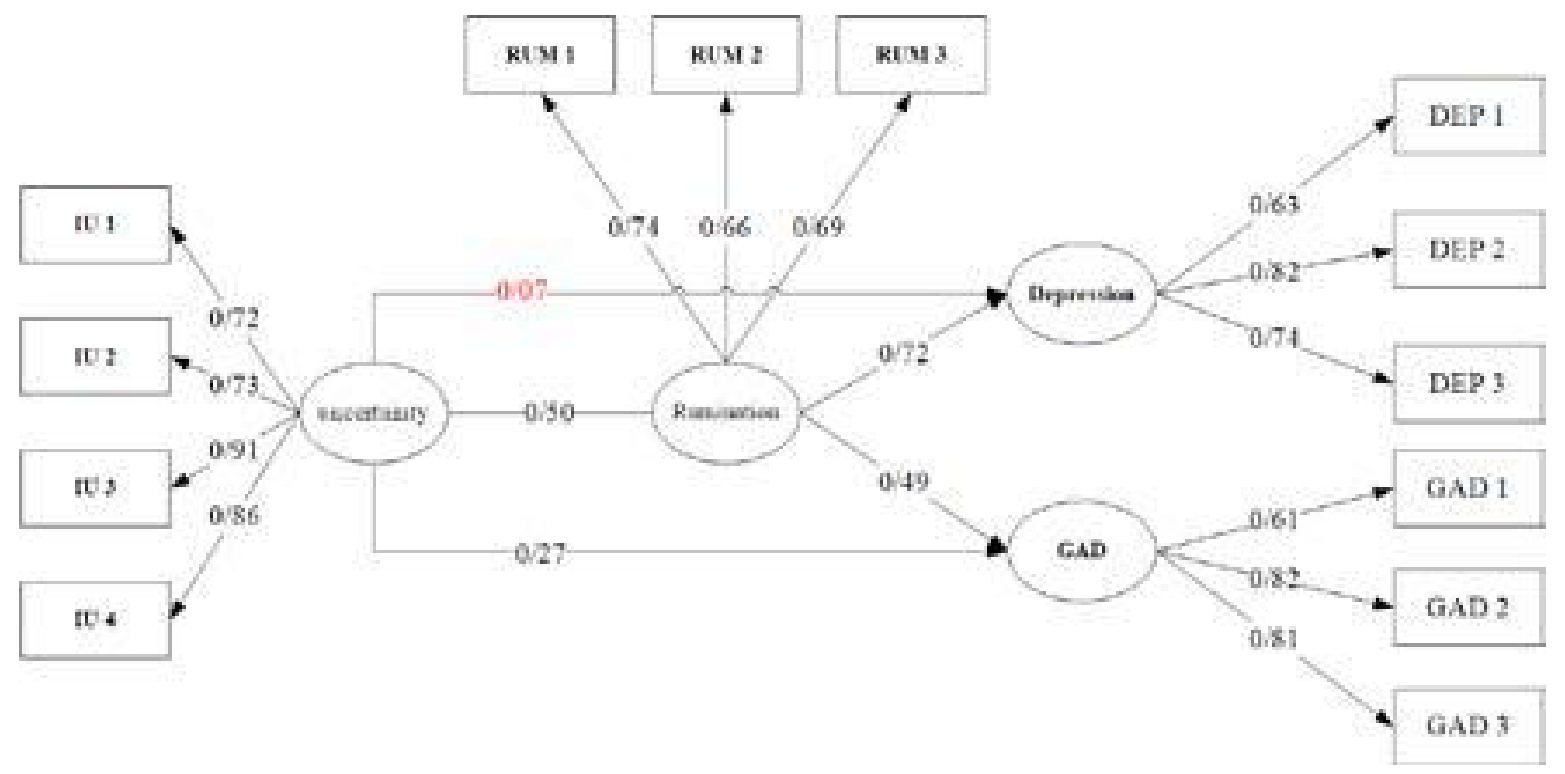

Figure 1. Structural model of the study and its standard coefficients 
concentration of rumination on negative experiences, which instead of active management, prolongs the stress associated with uncertainty intolerance. Perhaps, people with uncertain intolerance think that rumination will help them to better understand their feelings, gain insights and find solutions to the unknown situations they encounter.

\section{Ethical Considerations}

Compliance with ethical guidelines

All ethical principles were considered in this article. The participants were informed about the purpose of the research and its implementation stages; they were also assured about the confidentiality of their information; Moreover, They were allowed to leave the study whenever they wish, and if desired, the results of the research would be available to them.

\section{Funding}

This research did not receive any specific grant from funding agencies in the public, commercial, or not-forprofit sectors.

\section{Authors contributions}

All authors contributed in preparing this article.

\section{Conflict of interest}

The authors declare no conflict of interest. 
This Page Intentionally Left Blank 


\title{
مدلسازى معادلههاى ساختارى تحملنكردن بلاتكليفى و افسردتى و اختلال اضطراب فراتير: بررسى نقش واسطهاي نشخوارَ فِكرى
}

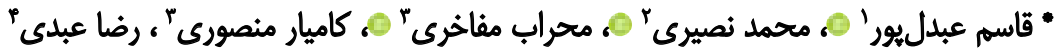

ا - دانشجوى دكتراى روانشناسى بالينى، كروه روانشناسى، دانشكده علوم انسانى، دانشغاه شاهد، تهران، ايران.

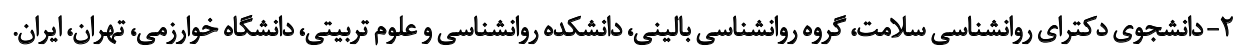

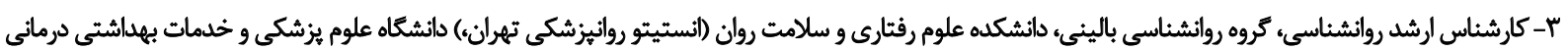
ايران، تهران، ايران.

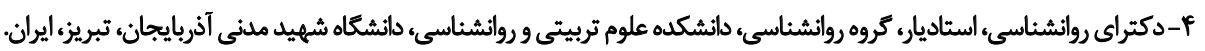

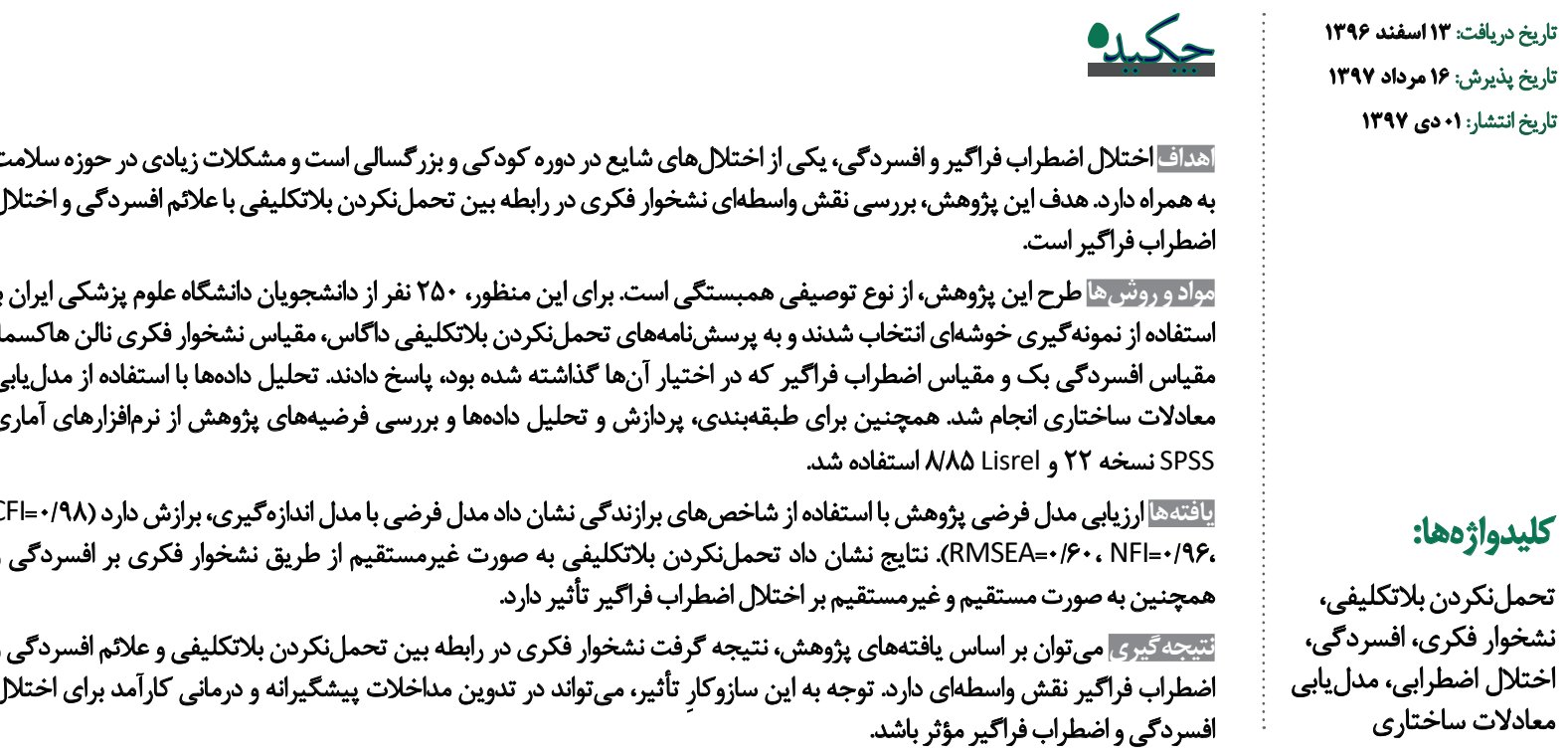

به اختلال اضطراب فراكير مبتلا هستند [F]]

dato

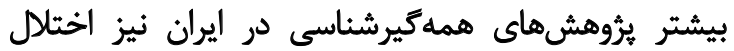

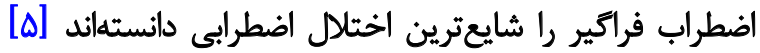

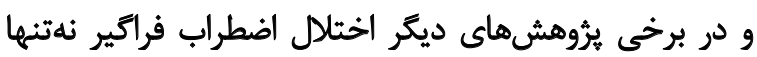

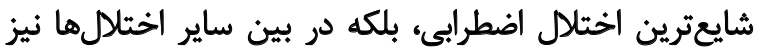

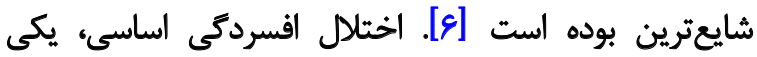

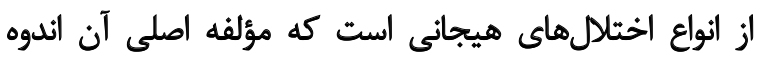

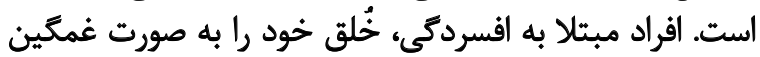

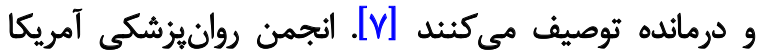

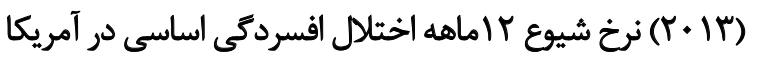

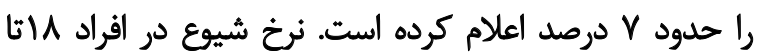

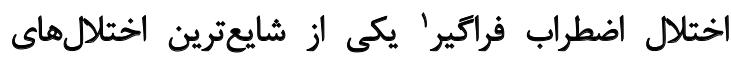

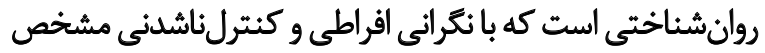

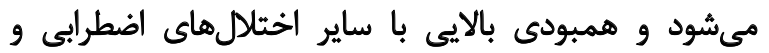

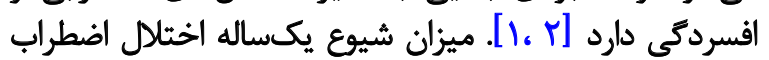

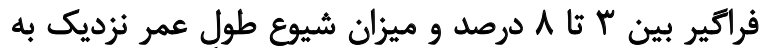

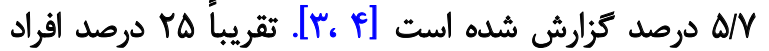

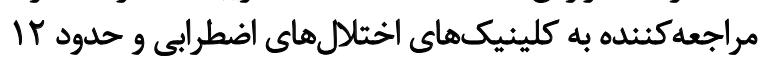

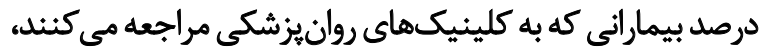

1. Generalized Anxiety Disorder (GAD)

* نويسئده مسئول:

قاسم عبدل ئور

نشاني: تهران، دانشعاه شاهده، دانشكده علوم انسانيء كروه روانشناسي. تلغن: يست الكترونيكى: gلين g.marand@yahoo.com 


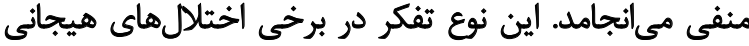

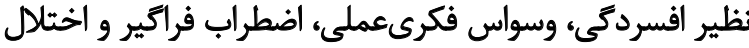

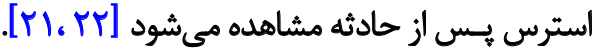

مدلها و تعاريف زيادى از نشخوار فكرى ارائه شده است

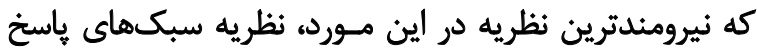

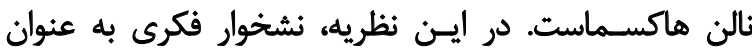

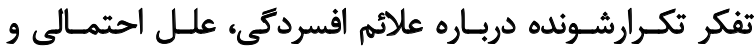

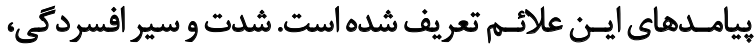

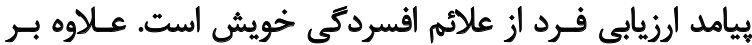

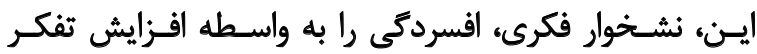

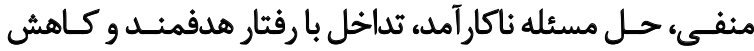

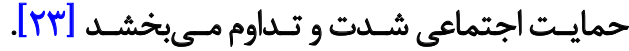
در مطالعـات متعـدد آزمايشى و مقطعى نيز نشان داده شد في

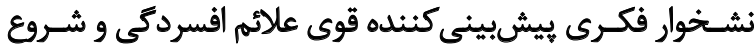

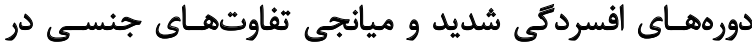

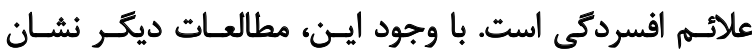

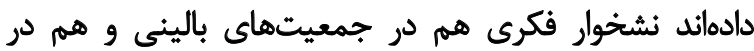

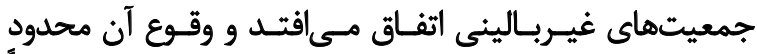

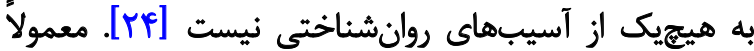

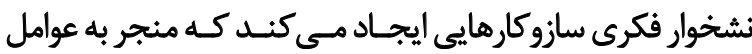

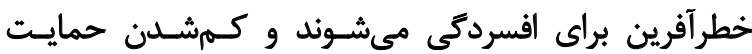

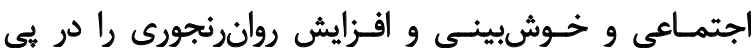

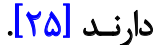

واتكينز و باراسيا بررسى كردند كه جرا افراد با وجود بيامدهاي

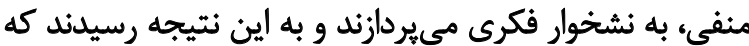

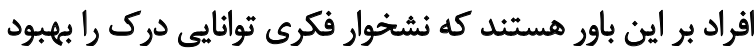

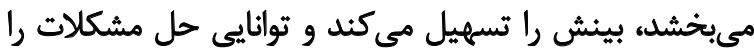

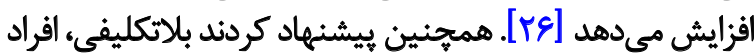

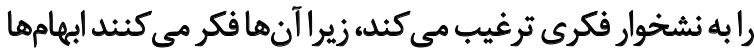

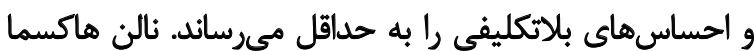

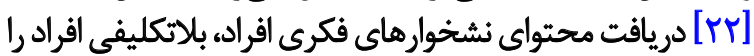

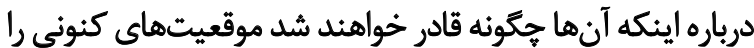

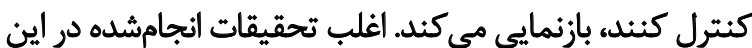

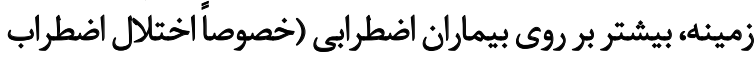

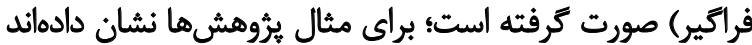

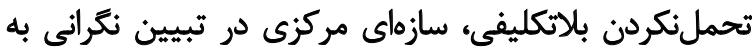

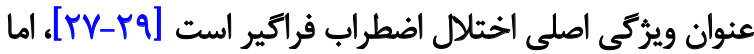

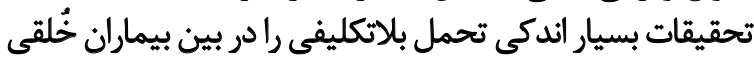

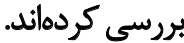

در يروهشى نشان داده شده است اختلال اضطراب فراكير با برديا

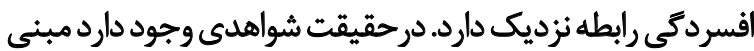

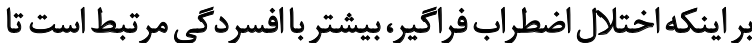

وباله، "ا برابر نرخ شيوع اين اختلال در افراد •وساله يا مسنتر

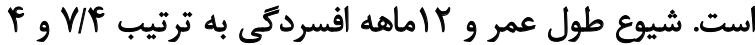

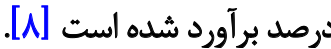

در ميان مؤلفههاى شناختى اضطراب فراكير، تحملنكردن ترني

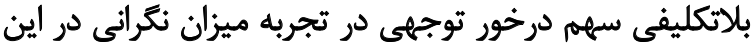

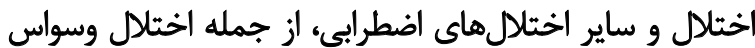

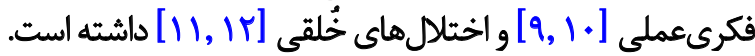

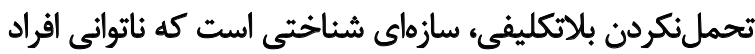

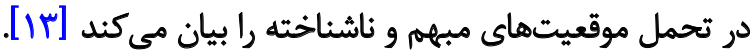

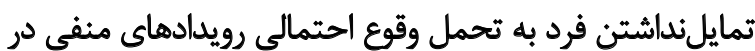

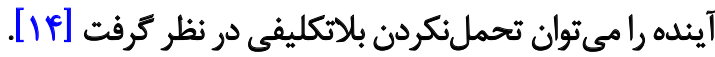
داكاس، باهر و لاداسر، تحملنكردن بلاتكليفى را سوكيرى

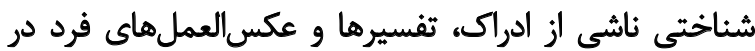

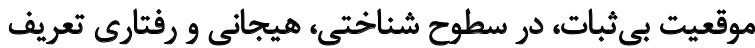

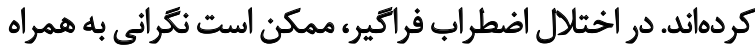

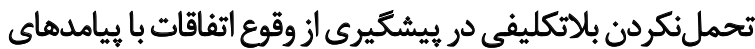

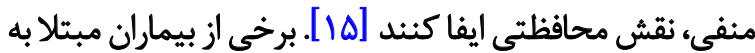

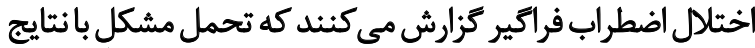

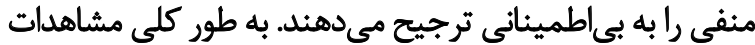

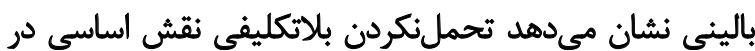

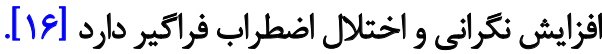

دجانك، بك و رايد، در يافتههاي خود نشان دادند افرادى كه

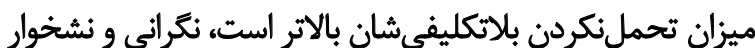

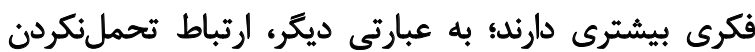

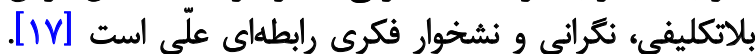

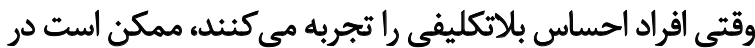

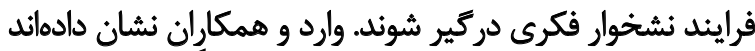

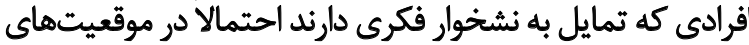

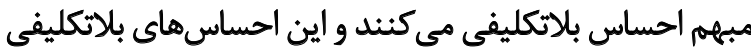

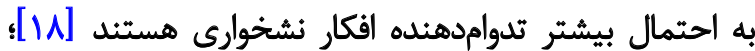

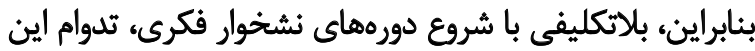

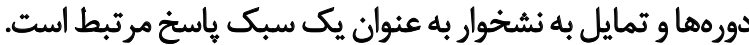

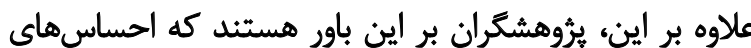

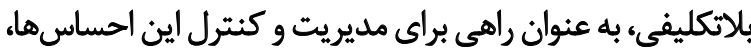
افراد رادر تداوم نشخوار فكرى قفل مي كنيند.

نشخوار فكرى، به طور كسترداى به عنوان تفكر تكرارى درباره

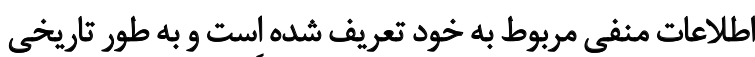

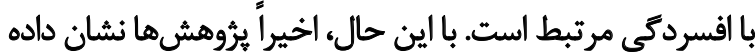

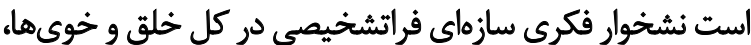

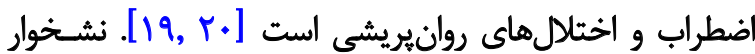

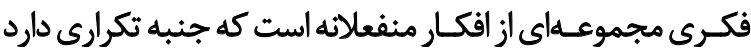

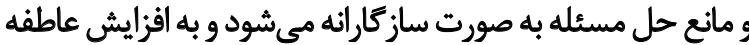


كردآورى دادههاء از نوع توصيفى همبستكى است. جامعه آمارى

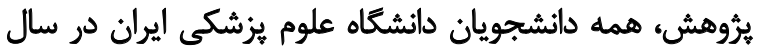

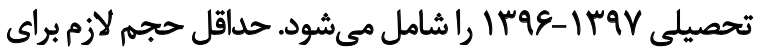

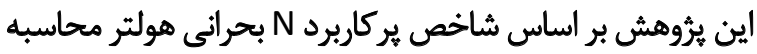

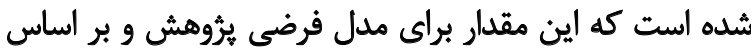

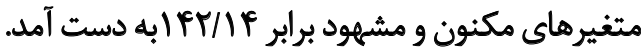

همجنينء با درنظركرفتن حداقل حجم موردنياز هنكامى كه

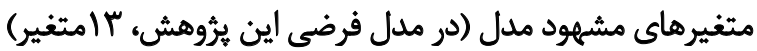

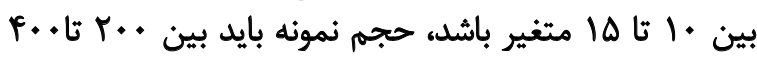

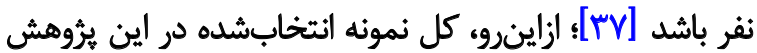

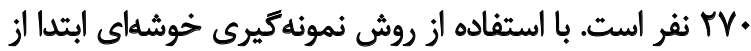

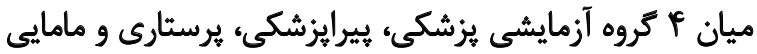

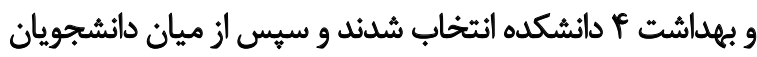

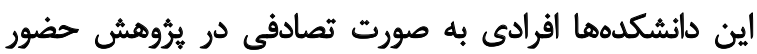

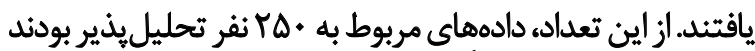

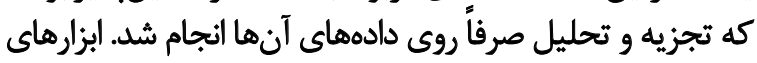

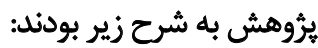

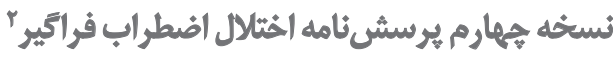

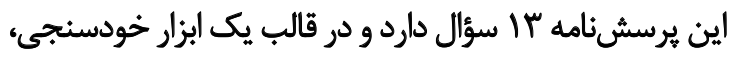

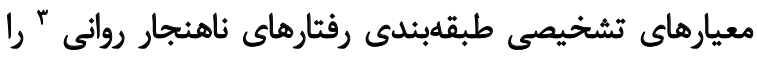

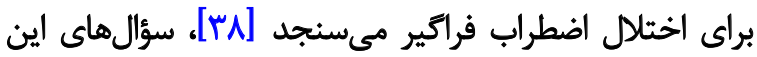

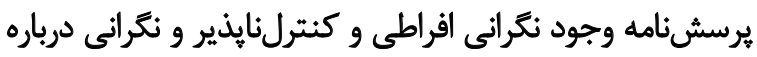

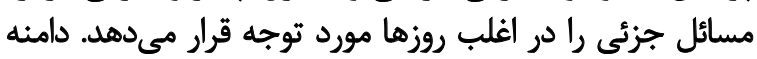

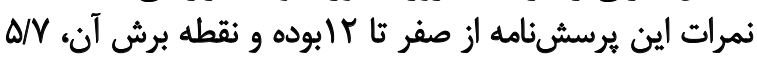

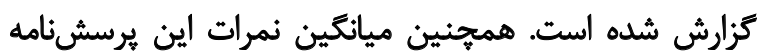

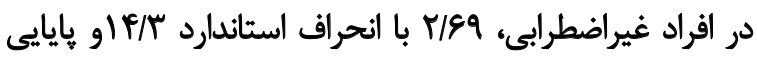

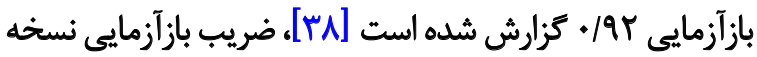

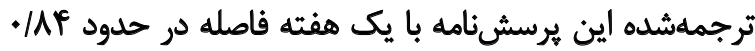

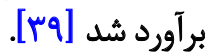

\section{مثياس تحمل تيكردن بلاتكليفي}

ايـن مقيـاس را فريستون و همكاران در سال 1997 الطراحي

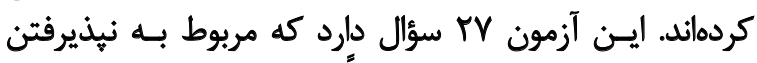

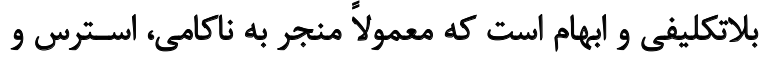

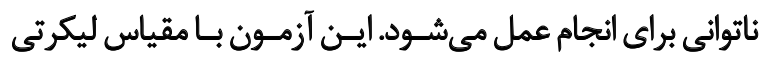

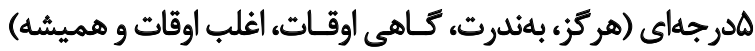

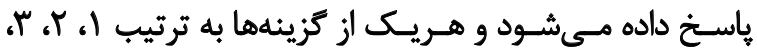

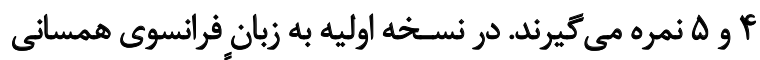

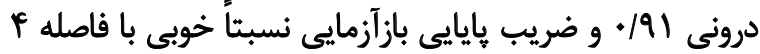

2. Generalized Anxiety Disorder Questionnaire-IV (GAD-Q-IV) 3. Diagnostic and Statistical Manual of Mental Disorders, $5^{\text {th }}$ Edition (DSM-IV)

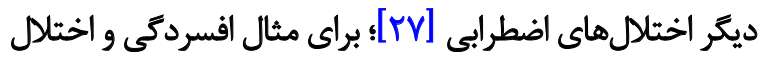

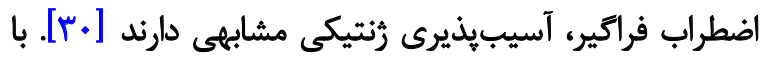

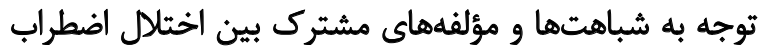

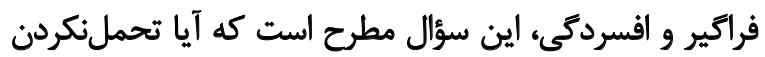

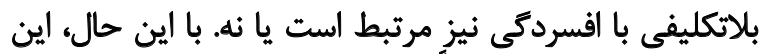

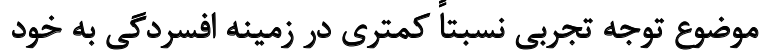

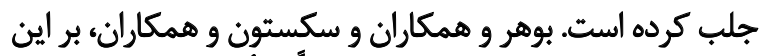

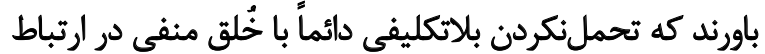

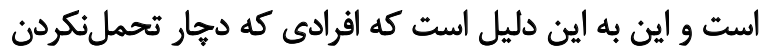

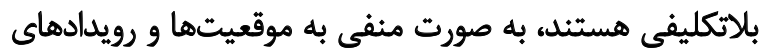

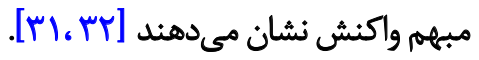

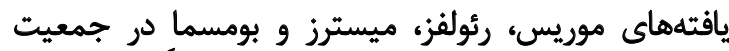

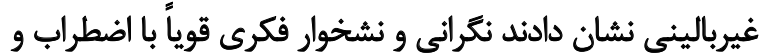

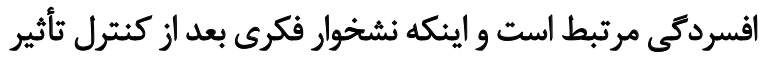

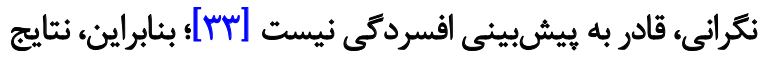

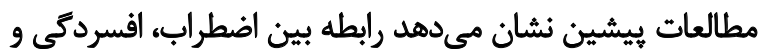

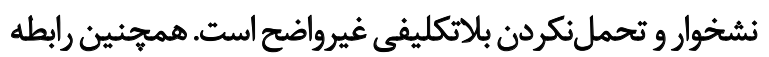

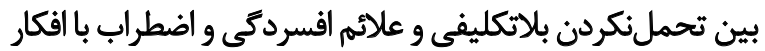

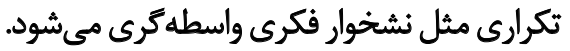

از لحاظ نظرى، يك ميانجى به عنوان متغير يا سازوكارى (مثل مئل

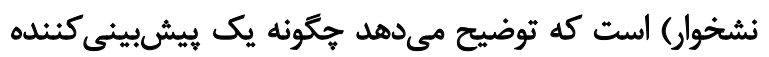

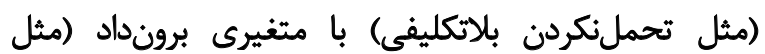

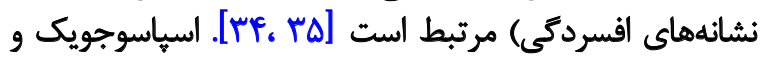

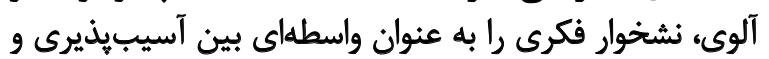

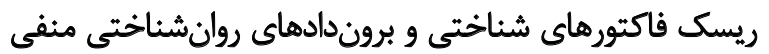

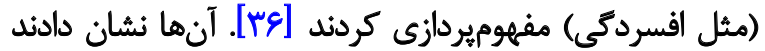

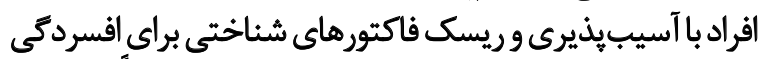

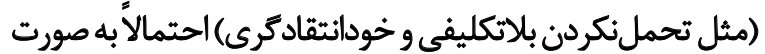

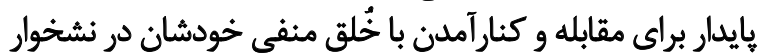

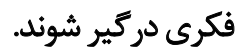

اسياسوجويك و آلوى بعدها توضيح دادند از آنجا كه نشخوار

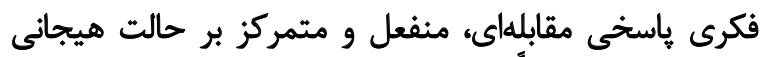

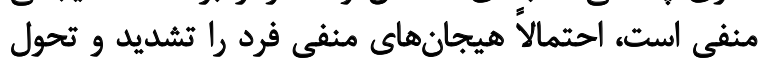

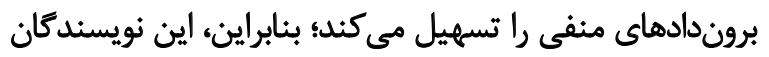

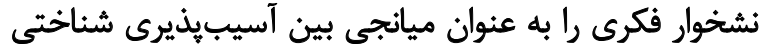

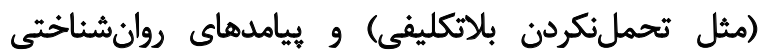

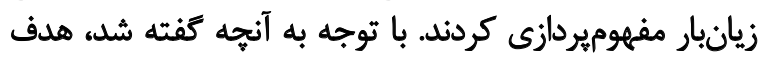

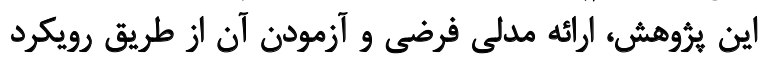

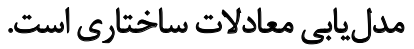
روش اين ثروهش به لحاظ هدف، از نوع بنيادى و به لحاظ نحوه 


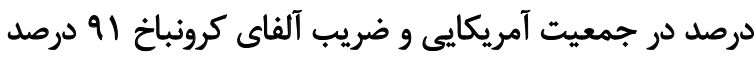

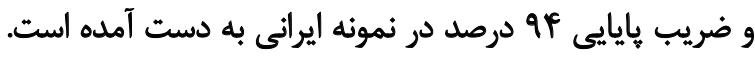

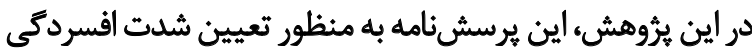

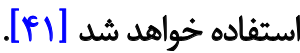

به منظور طبقهبندى، يردازش و تحليل دادهها و بررسى فرضيههاى ثرؤهش از نرمافزارهاى آمارى LISREL

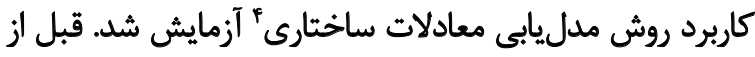

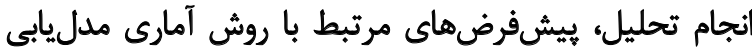

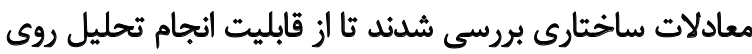

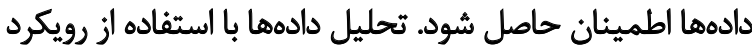

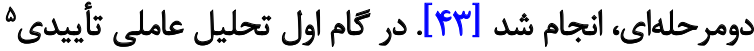

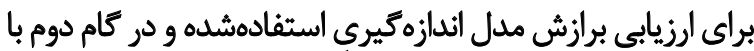

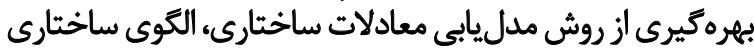

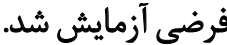

يافتهها

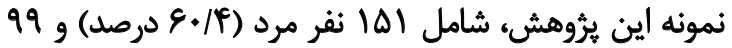

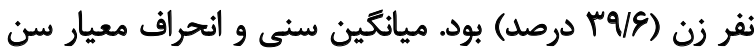

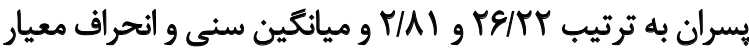

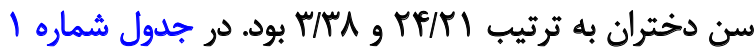

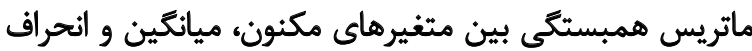

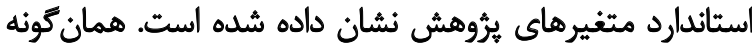

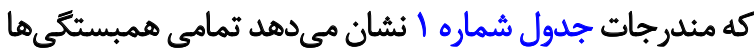
مقدار درخور توجهى را شامل مئشوند (

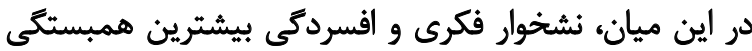

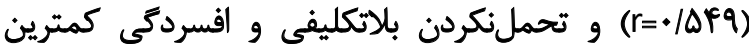

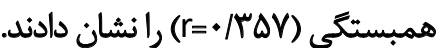

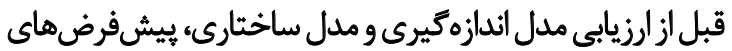

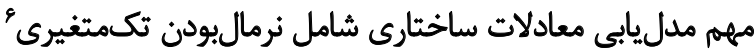

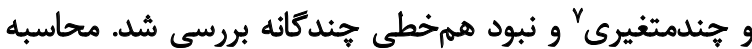

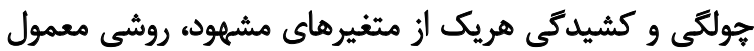

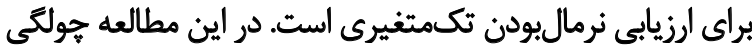

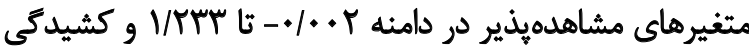

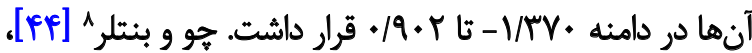

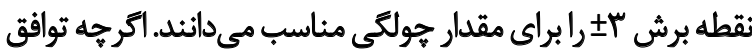

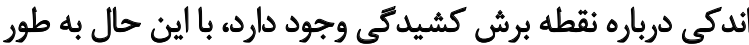

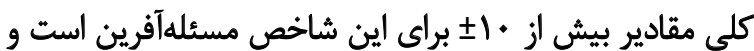

4. Structural Equation Modeling (SEM)

5. Confirmatory Factor Analysis (CFA)

6. Univariate normality

7. Multivariate normality

8. Chou \& Bentler

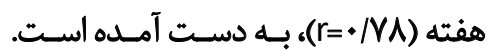

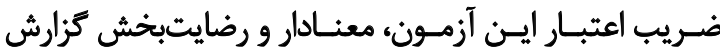

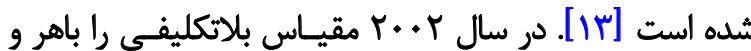

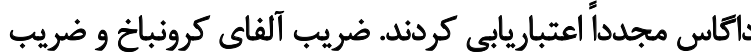

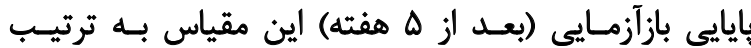

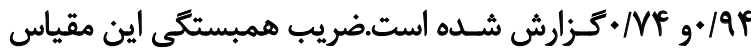

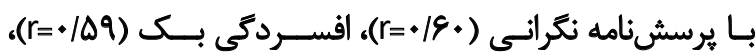

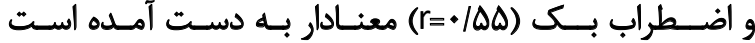

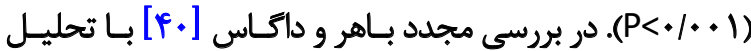

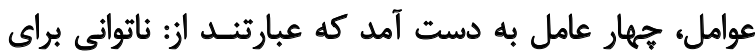

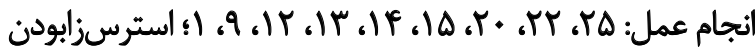

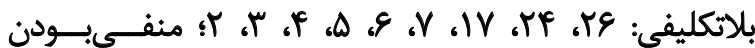

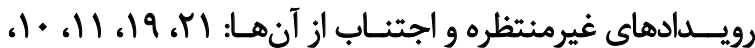

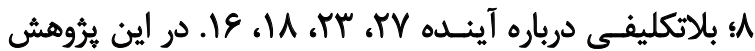
ضريب آلفاي كرونباخ نمره كل تحملنكردن بلاتكليفى 19 /.به

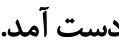

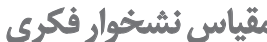

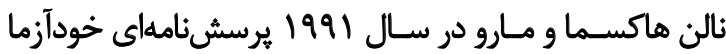

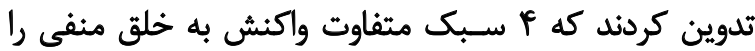

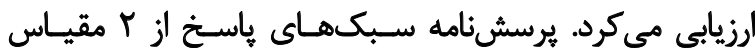

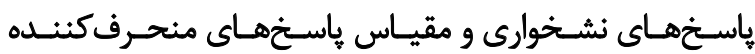

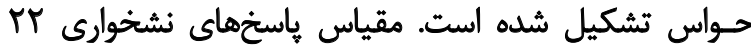

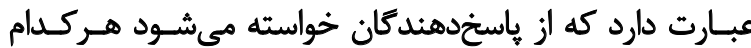

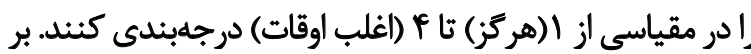

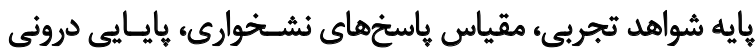

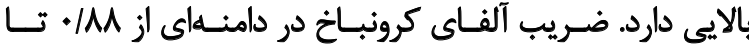

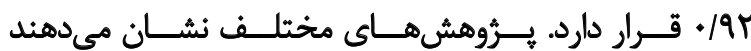

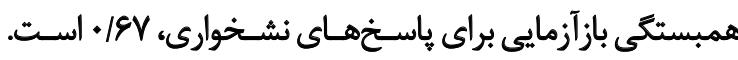

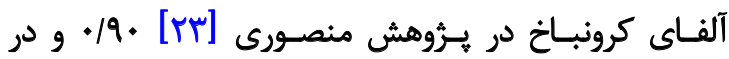

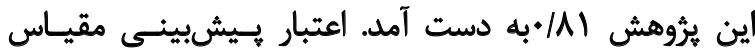

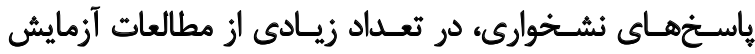

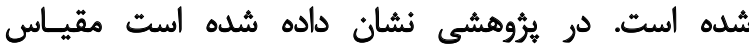

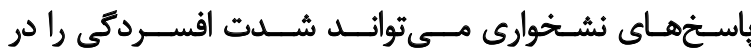

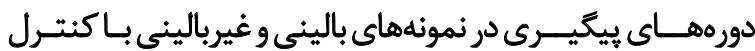

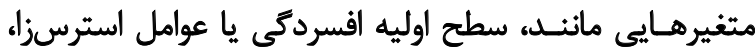

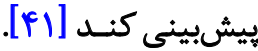

$$
\text { يرسش ثامه افسردمّى بك، وير ايش دوم }
$$

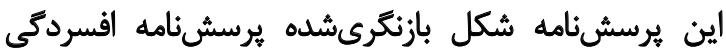

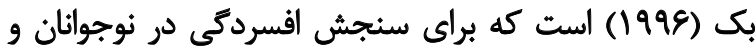

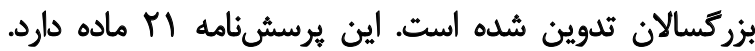

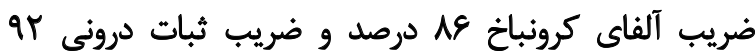


جدول ا. ماتريس همبستكى متغيرهاى يثروش، ميانكين و انحراف استاندارد

\begin{tabular}{|c|c|c|c|c|c|}
\hline مياتئين (انحراف اسثاندارد) & $\varepsilon$ & $r$ & $r$ & 1 & متغير هاى يثروهش \\
\hline$V / 41 \cdot \pm(N \cdot 91$ & & & & 1 & تحمل نكردن بلاتكليفى \\
\hline 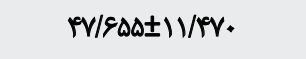 & & & 1 &.$/ 1 \cdot q^{* * *}$ & نشخوار فكرى \\
\hline $\mathbb{H} / A \Delta)^{\prime} \pm V / T \Delta \Delta$ & & 1 &.$/ \Delta p q * *$ & . rusures & الفسردمى \\
\hline $\mathrm{m} / \mathrm{R} \Delta \Delta \Delta / \mathrm{qI}$ & 1 & $. / p+r * *$ & . pera* & - pepren & اضطر|ب \\
\hline
\end{tabular}

جدول r. شاخصهاى برازش مدل اندازهيرى و مدل ساختارى بـروهش

\begin{tabular}{|c|c|c|c|c|c|c|c|c|}
\hline NFI & CFI & GFI & SRMR & RMSEA & $x^{2} / \mathrm{df}$ & df & Chi-Square & شاخصها \\
\hline .198 & .194 &.$/ 94$ & $.1 . \Delta f$ & .1 .09 & VEq & $\Delta q$ & $1 \ldots / r 1$ & مدل اندازهيرى \\
\hline .19 & .194 & . & $1+\Delta \Delta$ & .1 .9 & I/TYE & q. & $1 . r / 09$ & هدل ساختارى \\
\hline
\end{tabular}

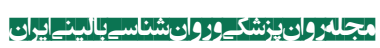

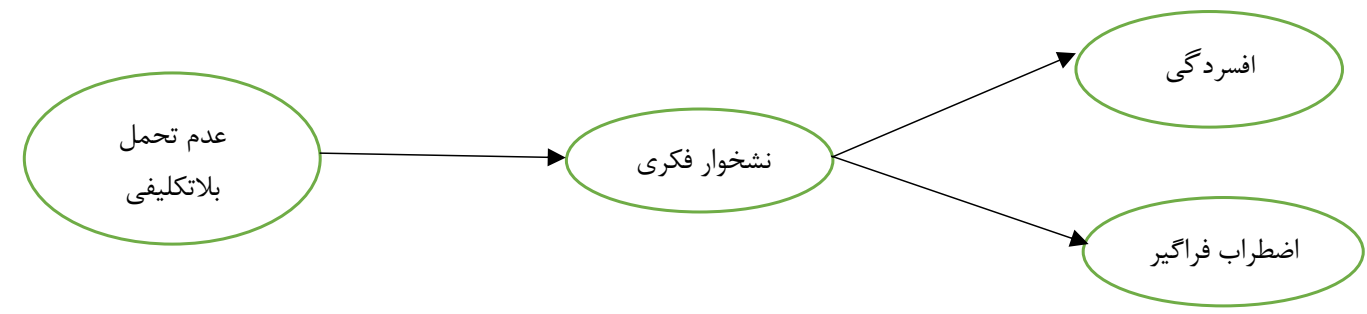

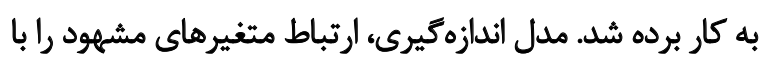

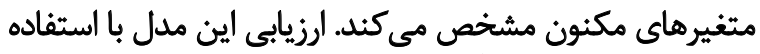

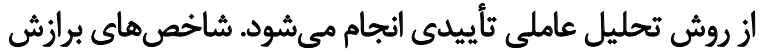

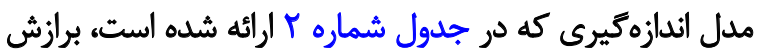

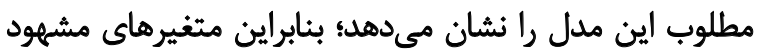

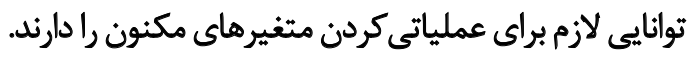

همجنين ارزيابي مدل ساختارى با استفاده از روش مدل يابي

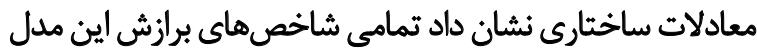

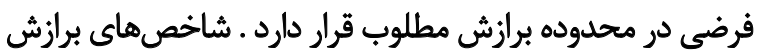

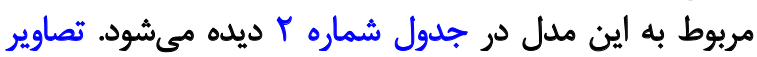

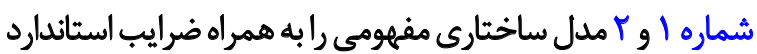

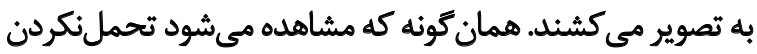

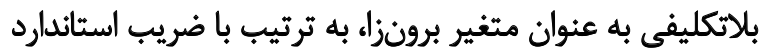

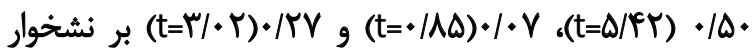

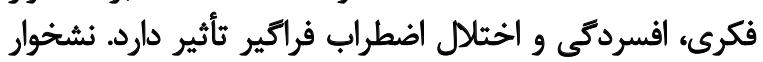

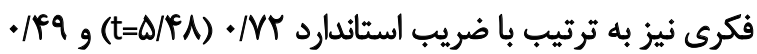
(t=F/fl)

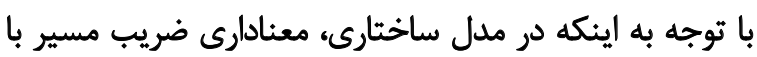

مقادير بيش از · بـ نتايج بهدستآمده رانامعتبر مي كند [FD]. در اين مطالعه فرض نرمالبودن جندمتغيرى با محاسبه

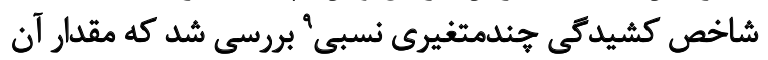

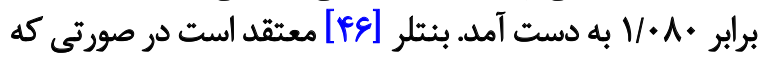

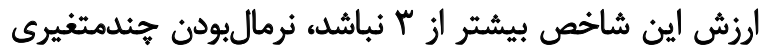

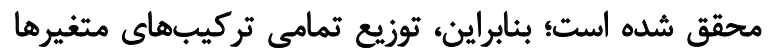

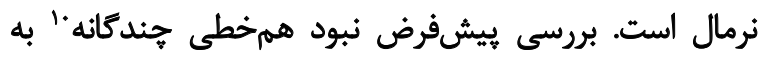
وسيله وارسي ماتريس همبستئي بين متغير هاي مشهرود نيود انجام

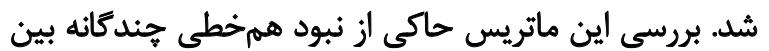

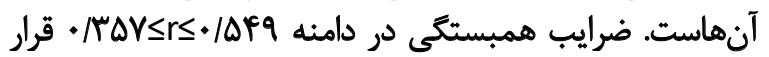

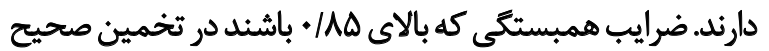

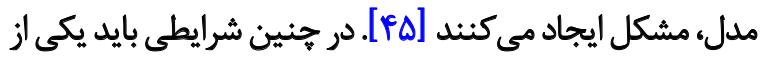
دو متغير از تحليل كنار كذاشته شود.

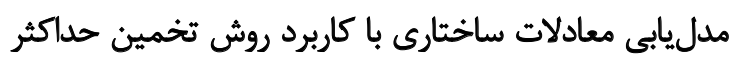

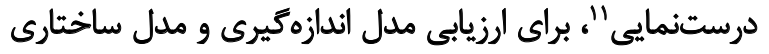

9. Relative multivariate kurtosis

10. Multicollinearity

11. Maximum likelihood 
جدول ٪. نتايج آزمون بوثاسترب براي روابط واسطهاي

\begin{tabular}{|c|c|c|c|c|c|c|c|}
\hline \multirow{2}{*}{ سطح معنى دارى } & \multirow{2}{*}{ اندازه اثث } & \multirow{2}{*}{ خطاي برآورد } & \multicolumn{2}{|c|}{ حدود بوت|سترب } & \multirow{2}{*}{ هتغير وابسته } & \multirow{2}{*}{ متغير واسط } & \multirow{2}{*}{ متغير مسثقل } \\
\hline & & & حد بايين & حد بالا & & & \\
\hline $.1 . \Delta$ &.$/ M V$ & .1 .89 & $\cdot \pi \cdot \Delta$ &.$/ 589$ & افسردمى & نُشَخوار فكرى & تحملنكردن بالتكليفى \\
\hline$\cdot 1 \cdot \Delta$ &.$/ M I E$ & $.1 . \Delta A$ & $.11 \cdot r$ &.$/ M T q$ & GAD & نشختوار فكرى & تحملنكردن بلاتكليفي \\
\hline
\end{tabular}

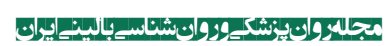

مسير تحملنكردن بلاتكليفى به اضطراب فراكير با واسطه

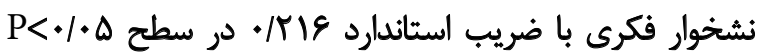

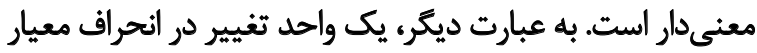

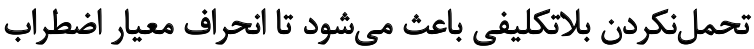

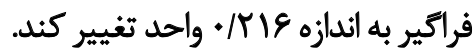

$\stackrel{\leftrightarrow}{\leftrightarrow}$

هدف از اين مطالعه، بررسى نقش واسطهاي نشخوار فكرى در

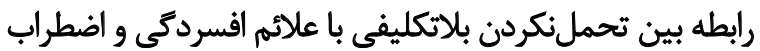

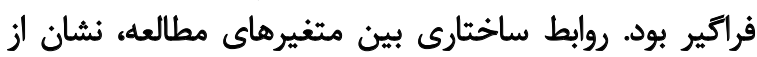

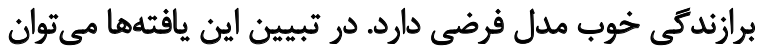

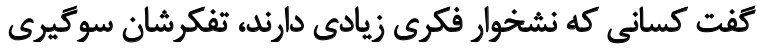

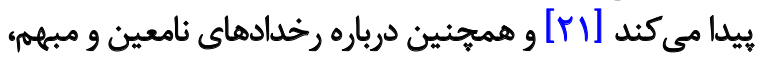

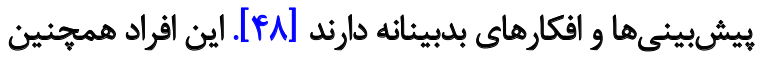
ممكن است دسترسى سريع به خاطرات منفى داشته باشين باشند كه با بانيا

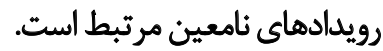

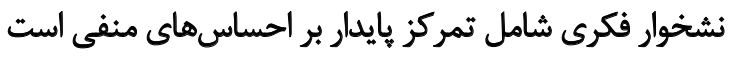

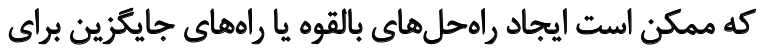

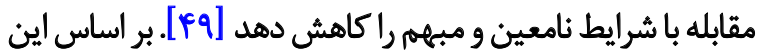

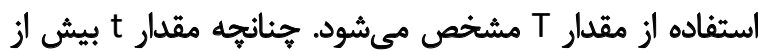

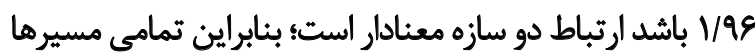

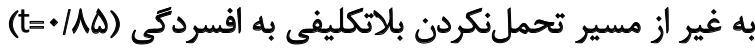

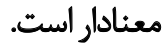

در اين مطالعه براى ارزيابى روابط واسطهايى از آزمون

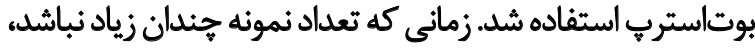

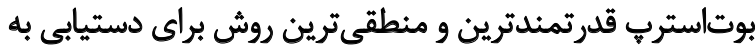

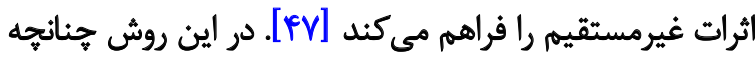

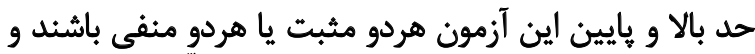

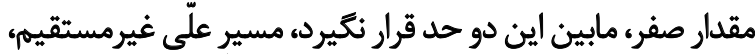

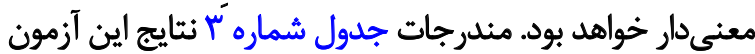

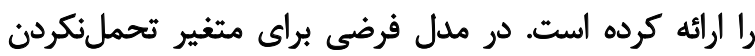

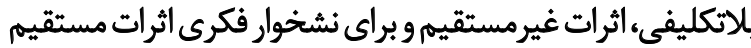
در نظر كرفته شده بودي

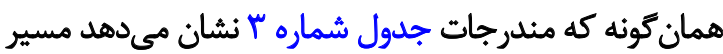

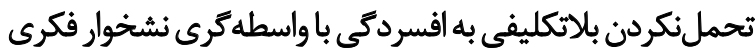

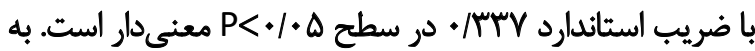

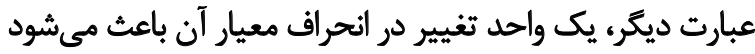

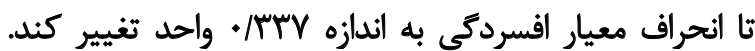

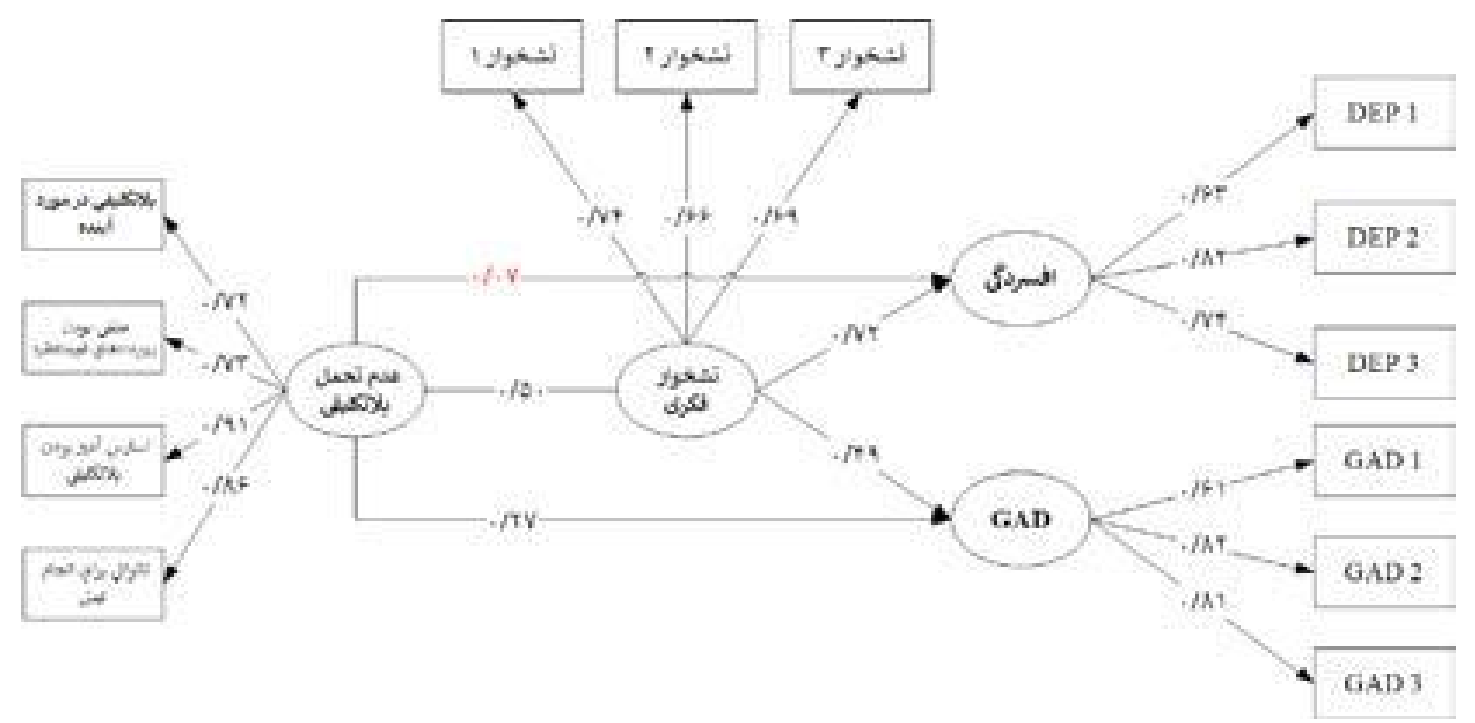




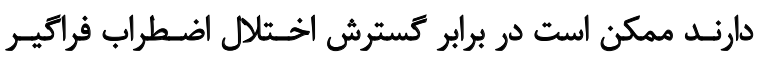

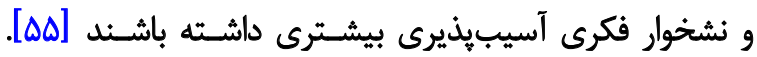

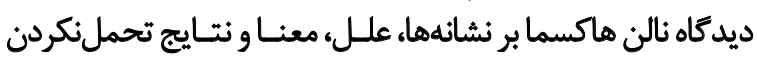

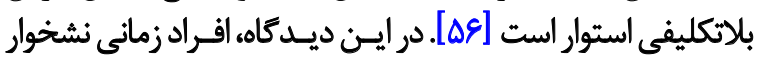

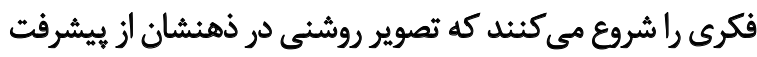

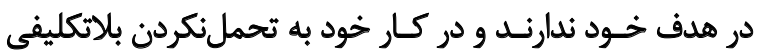

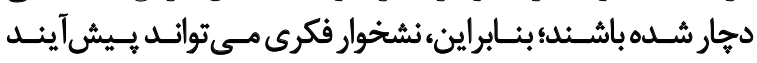

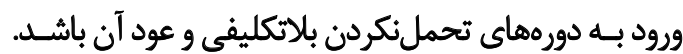

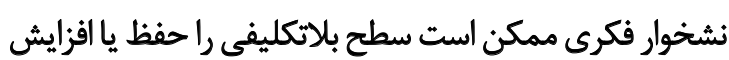

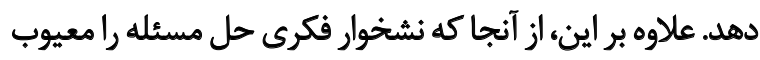

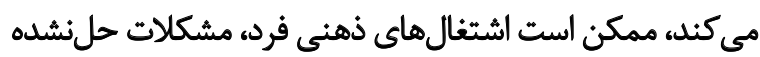

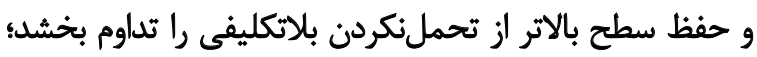

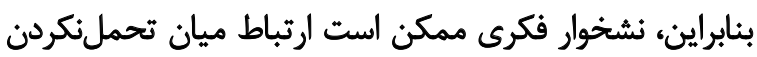

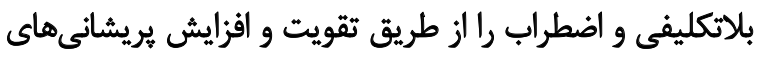

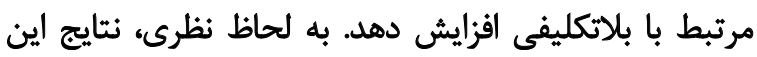

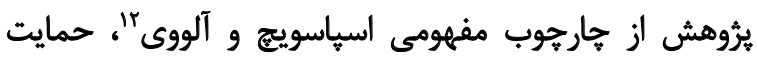

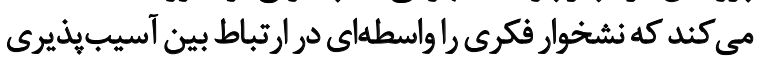

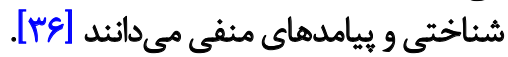

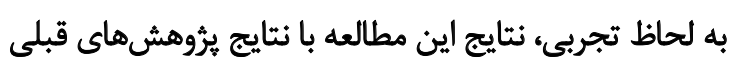

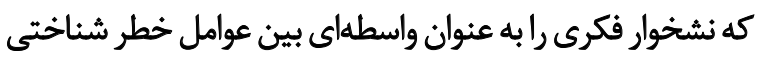

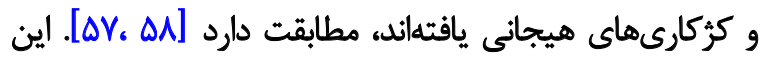

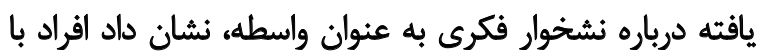

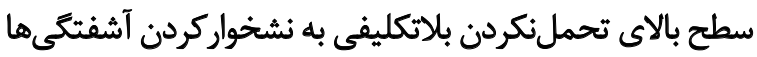

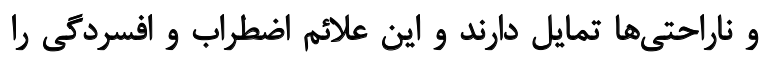
كستردةترمى كند.

نشخوار فكرى راهبردهاى مقابلهاي ناسازكارانه براى كنترل و و

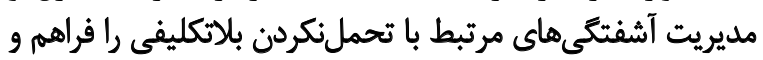

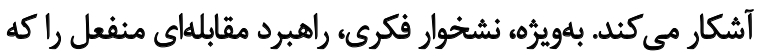

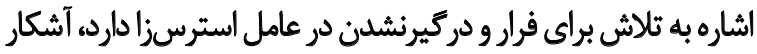

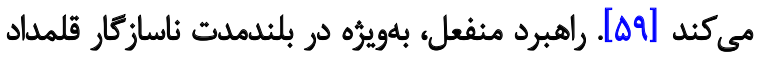

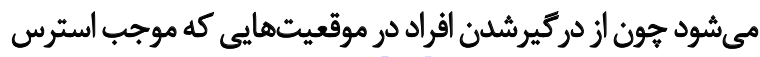

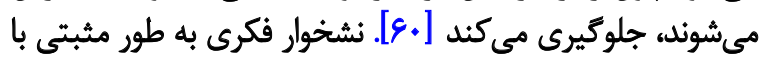

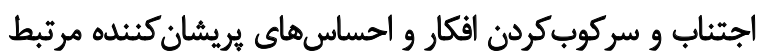

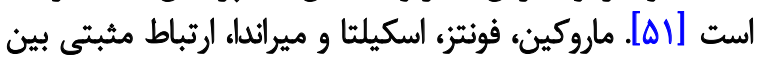

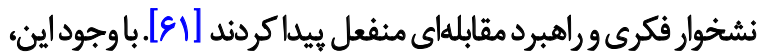

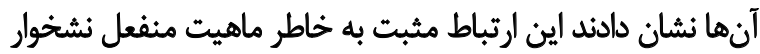

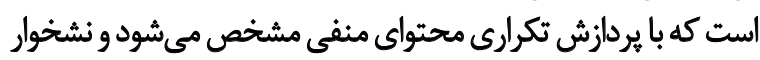
را به نشانههاى افسرد بكى مرتبط مي كنئد

در اين مطالعه، تحملنكردن بلاتكليفى ممكن است منجر به به

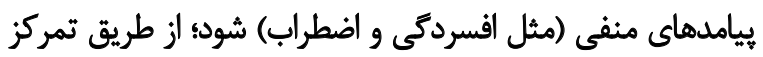

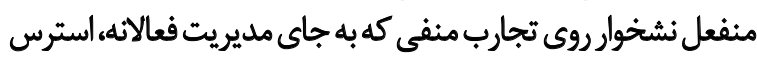

12. Spasojevic \& Alloy
بيامدهاى منفى نشخوار فكرى، احتمالاً نشخوار فكرى رابطه بين

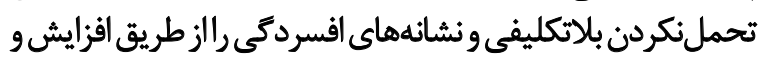

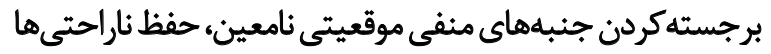

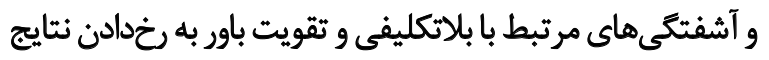

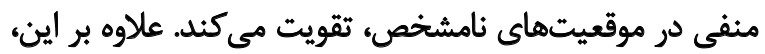

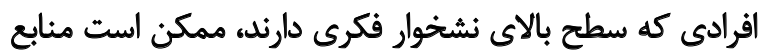

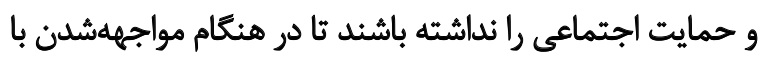

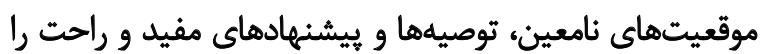

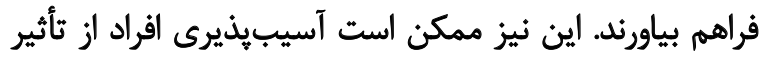

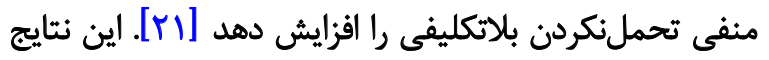

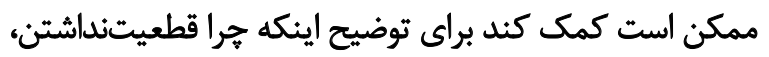

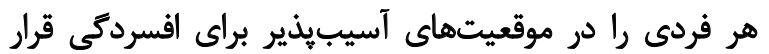

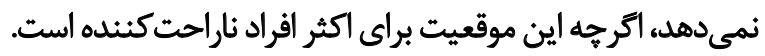

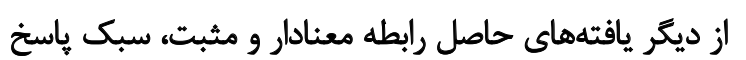

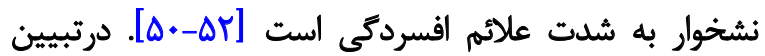

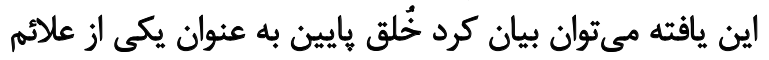

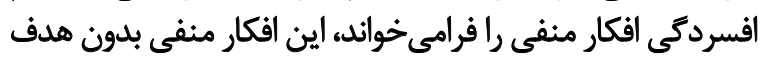

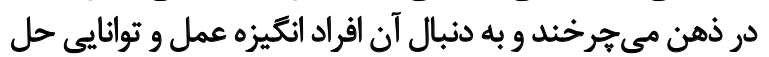

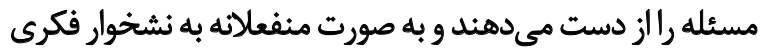

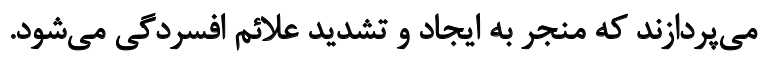

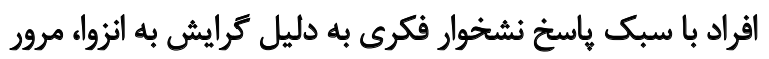

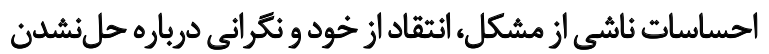

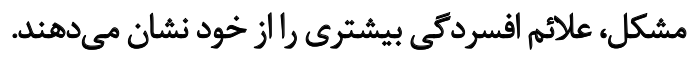

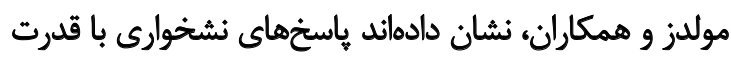

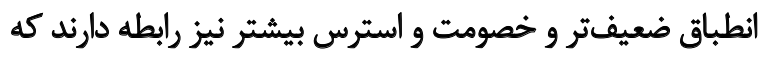

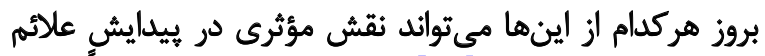

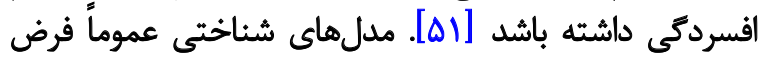

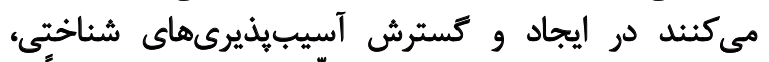

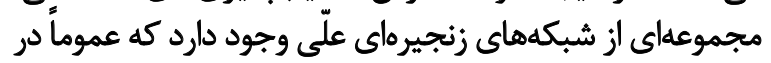

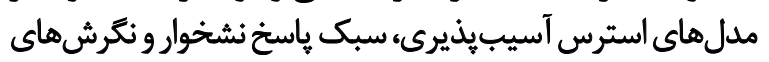

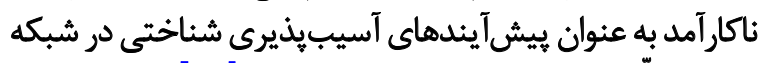

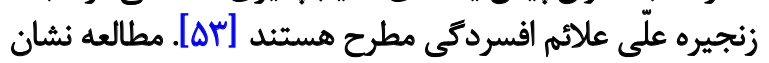

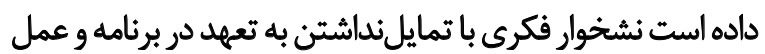

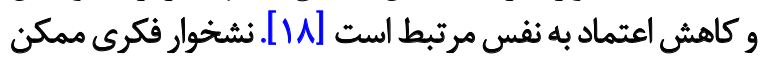

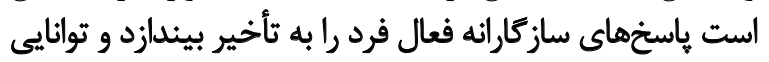

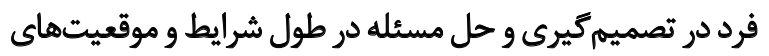

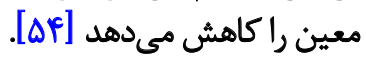

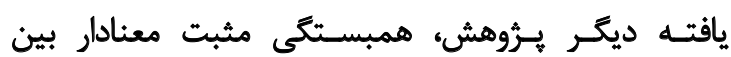

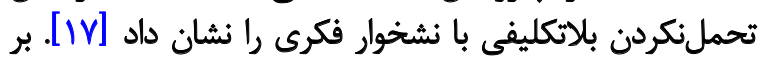

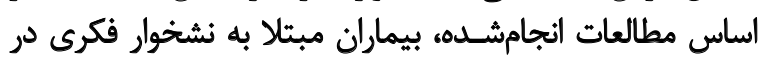

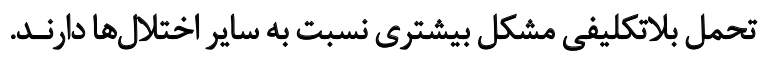

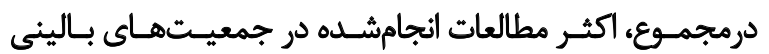

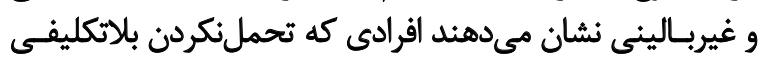


است كه جمعيت نسبتاً همكنى هستند؛ بنابراين، در تعميم

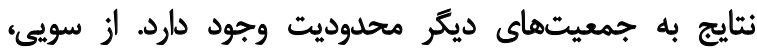

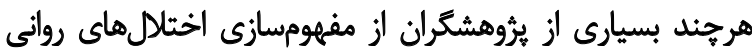

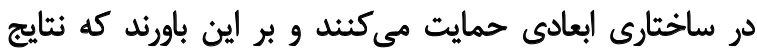

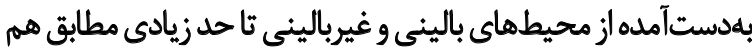

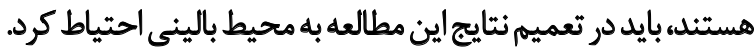
محدوديتديكراينكهماهيت مقطعى اينمطالعه، مانعاستنتاجهاى

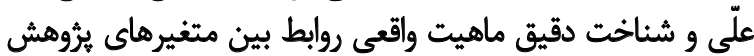

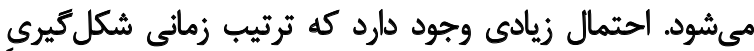

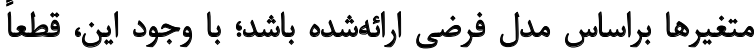

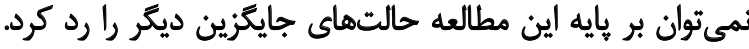

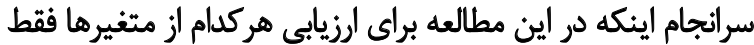

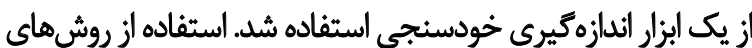

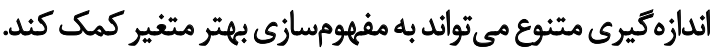

با توجه به محدوديتهاى يزووهش، يُيشنهاد مىشود اين مطالعه

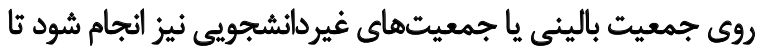

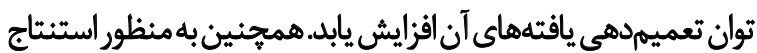

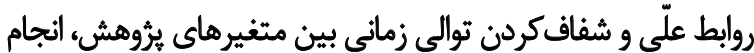

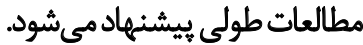

ملاحظات اخلاقي

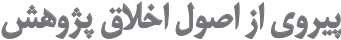

همه اصول اخلاقى در اين مقاله رعايت شده است. شركت كنيندكان

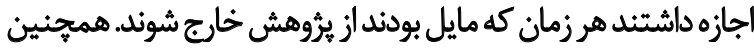

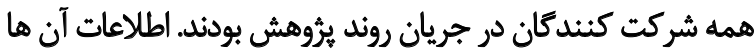

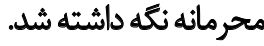

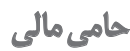

اين مقاله حامى مالى نداشته است.

مشار كتونويسندكان

تمام نويسندكان در آمادهازى اين مقاله مشاركت داشتهاند.

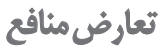

بنابر اظهار نويسندكان، اين مقاله تعارض منافع ندارد.
مربوط به تحمل نكردن بلاتكليفى راطولانى تر مي كند. احتمالاً، افراد

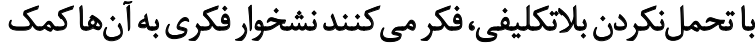

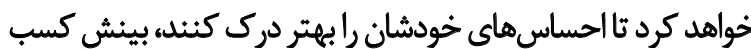

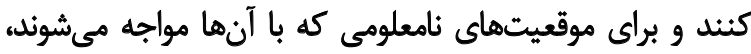

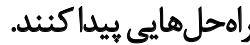
از ديكر يافتهاى اين بثروهش، رابطه مستقيم معنادار بين

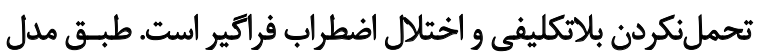

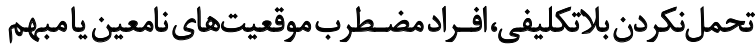

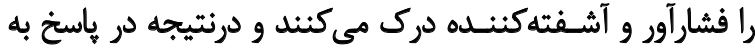

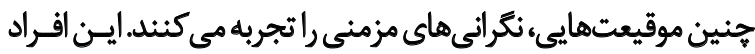

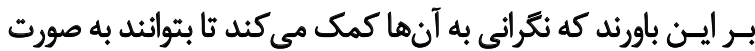

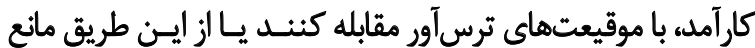
وقوع جنين حوادثى شوند.

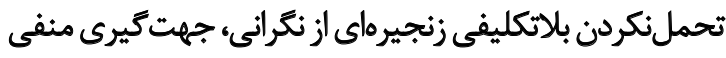

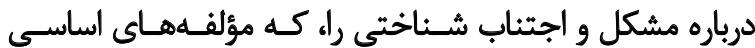

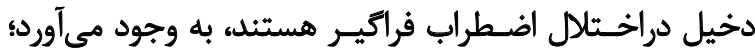

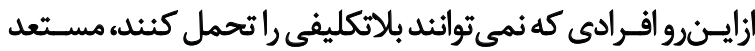

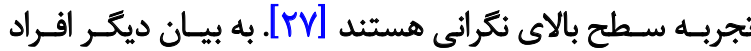

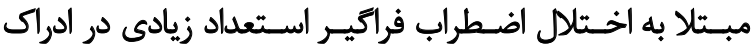

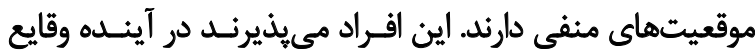

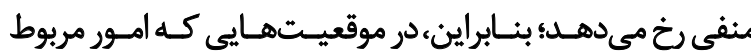

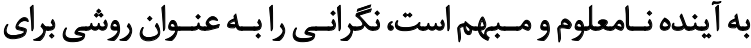

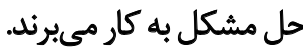

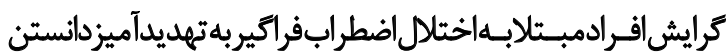

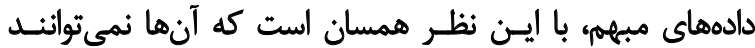

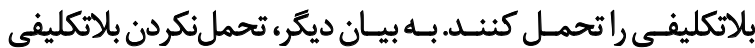

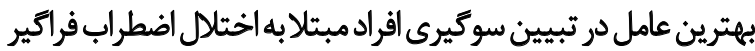

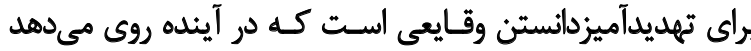

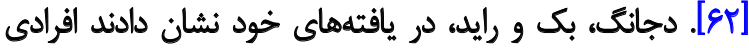

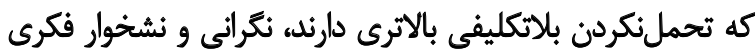

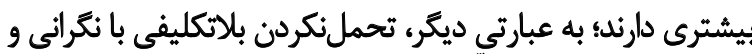

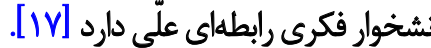

$$
\text { تشيجهيرى }
$$

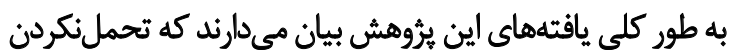

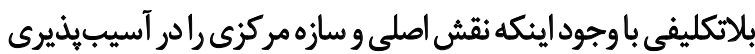

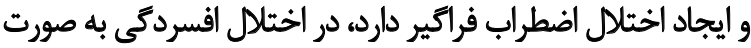

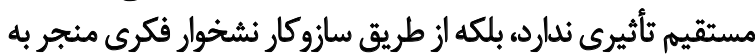

$$
\text { افسردكى مي مشيود. }
$$

با اينكه يافتهها از مدل فرضى ثيروهش حمايتكردند، نتايج

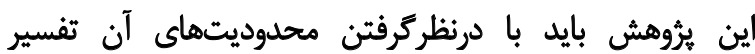
شود. ابتدا اينكه اين مطالعه روى داين دانشجويان انجام شده اند تفير 


\section{References}

[1] Sadock BJ, Sadock VA. Kaplan and Sadock's synopsis of psychiatry: Behavioral sciences/clinical psychiatry. Philadelphia: Lippincott Williams \& Wilkins; 2011.

[2] Shihata S, McEvoy PM, Mullan BA. Pathways from uncertainty to anxiety: An evaluation of a hierarchical model of trait and disorder-specific intolerance of uncertainty on anxiety disorder symptoms. Journal of Anxiety Disorders. 2017; 45:72-9. [DOI: 10.1016/j.janxdis.2016.12.001] [PMID]

[3] American Psychiatric Association. Diagnostic and Statistical Manual of Mental disorders (DSM-5). Washington: American Psychiatric Association; 2013.

[4] Kessler R, Walters E, Wittchen HU. Epidemiology. In: Heimberg RG, Turk CL, Mennin DS, editors. Generalized Anxiety Disorder: Advances in Research and Practice. New York: Guilford Press; 2004.

[5] Ehsanmanesh M. [Epidemiology of mental disorders in Iran (Persian)]. Iranian Journal of Psychiatry \& Clinical Psychology. 2001; 6(4):54-70.

[6] Omidi A, Tabatabai A, Sazvar A, Akkashe G. [Epidemiology of mental disorders in urbanized areas of Natanz (Persian)]. Iranian Journal of Psychiatry \& Clinical Psychology. 2003; 8(4):32-8.

[7] Brain C. Advance psychology: Application, issues and perspective. Cheltenham: Nelson Thorenes; 2002.

[8] Al Hamzawi AO, Bruffaerts R, Bromet EJ, AlKhafaii AM, Kessler $\mathrm{RC}$. The epidemiology of major depressive episode in the Iraqi general population. PLOS One. 2015; 10(7):e0131937.

[9] Boelen PA, Reijntjes A. Intolerance of uncertainty and social anxiety. Journal of Anxiety Disorders. 2009; 23(1):130-5

[10] Agheli F, Hasanzadeh R, Ghasemian D. [Compare of intolerance of uncertainty among obsessive-compulsive disorder, generalized anxiety disorder and healthy subjects (Persian)]. Journal of Novel Applied Sciences. 2013; 2(2):896-9.

[11] Buhr K, Dugas MJ. Investigating the construct validity of intolerance of uncertainty and its unique relationship with worry. Journal of Anxiety Disorders. 2006; 20(2):222-36.

[12] Dugas MJ, Robichaud M. Cognitive-behavioral treatment for generalized anxiety disorder: From science to practice. New York: Routledge; 2007.

[13] Freeston MH, Rhéaume J, Letarte H, Dugas MJ, Ladouceur R. Why do people worry? Personality and Individual Differences. 1994; 17(6):791-802. [DOI:10.1016/0191-8869(94)90048-5]

[14] Holaway RM, Heimberg RG, Coles ME. A comparison of intolerance of uncertainty in analogue obsessive-compulsive disorder and generalized anxiety disorder. Journal of Anxiety Disorders. 2006; 20(2):158-74.

[15] Dugas MJ, Buhr K, Ladouceur R. The role of intolerance of uncertainty in the etiology and maintenance of generalized anxiety disorder. In: Heimberg RG, Turk CL, Mennin DS, editors. Generalized Anxiety Disorder: Advances in Research and Practice. New York: Guilford Press; 2003.

[16] Heimberg RG, Turk CL, Mennin DS. Generalized anxiety disorder: Advances in research and practice. New York: Guilford Press; 2004.
[17] de Jong Meyer R, Beck B, Riede K. Relationships between rumination, worry, intolerance of uncertainty and metacognitive beliefs. Personality and Individual Differences. 2009; 46(4):547-51. [DOI:10.1016/j.paid.2008.12.010]

[18] Ward A, Lyubomirsky S, Sousa L, Nolen Hoeksema S. Can't quite commit: Rumination and uncertainty. Personality and Social Psychology Bulletin. 2003; 29(1):96-107. [DOI:10.1177/0146167202238375]

[19] Hartley S, Haddock G, e Sa DV, Emsley R, Barrowclough C. An experience sampling study of worry and rumination in psychosis. Psychological Medicine. 2014; 44(8):1605-14. [DOI:10.1017/ S0033291713002080] [PMID]

[20] Wolkenstein L, Zwick JC, Hautzinger M, Joormann J. Cognitive emotion regulation in euthymic bipolar disorder. Journal of Affective Disorders. 2014; 160:92-7. [DOI:10.1016/j.jad.2013.12.022 ] [PMID]

[21] Nolen Hoeksema S, Davis CG. Thanks for sharing that: Ruminators and their social support networks. Journal of Personality and Social Psychology. 1999; 77(4):801. [DOI:10.1037/00223514.77.4.801] [PMID]

[22] Nolen Hoeksema S. The role of rumination in depressive disorders and mixed anxiety/depressive symptoms. Journal of Abnormal Psychology. 2000; 109(3):504-11. [DOI:10.1037/0021843X.109.3.504]

[23] Mansouri A, Farnam AR, Bakhshipour Rodsari A, MahmoodAliloo M. [Comparisons between rumination in Individual with major depresion disorder, obsessive-compulsive disorder. General Anxiety Disorder and normal persons (Persian)]. Journal of Sabzevar University of Medical Sciences. 2010; 4:26-9.

[24] Pearson M, Brewin CR, Rhodes J, McCarron C. Frequency and nature of rumination in chronic depression: A preliminary study. Cognitive Behaviour Therapy. 2008; 37(3):160-8. [DOI:10.1080/16506070801919224] [PMID]

[25] Papageorgiou C, Wells A. Positive beliefs about depressive rumination: Development and preliminary validation of a selfreport scale. Behaviour Therapy. 2001; 32(1):13-26.

[26] Watkins E, Baracaia S. Why do people ruminate in dysphoric moods? Personality and Individual Differences. 2001; 30(5):723-34.

[27] Dugas MJ, schewartz A, Francis K. Intolerance of uncertainty, worry, and depression. Cognitive Therapy and Research. 2004; 28(6):835-42. [DOI:10.1007/s10608-004-0669-0]

[28] Ladouceur R, Gosselin P, Dugas MJ. Experimental manipulation of intolerance of uncertainty: A study of a theoretical model of worry. Behaviour Research and Therapy. 2000; 38(9):933-41.

[29] Grenier S, Ladouceur R. [Manipulation de Intolerance al Incertitude et Inquietudes (French)]. Revue Comedienne Dessciences du Comportment. 2004; 36(1):56-65.

[30] Kendler KS. Major depression and generalised anxiety disorder: same genes, (partly) different environments-revisited. Focus. 2004; 168(3):68-425.

[31] Buhr K, Dugas MJ. The intolerance of uncertainty scale: Psychometric properties of the English version. Behaviour Research and Therapy. 2002; 40(8):931-45. 
[32] Sexton KA, Norton PJ, Walker JR, Norton GR. Hierarchical model of generalized and specific vulnerabilities in anxiety. Cognitive Behaviour Therapy. 2003; 32(2):82-94

[33] Muris P, Roelofs J, Meesters C, Boomsma P. Rumination and worry in nonclinical adolescents. Cognitive Therapy and Research. 2004; 28(4):539-54.

[34] Kraemer HC, Stice E, Kazdin A, Offord D, Kupfer D. How do risk factors work together? Mediators, moderators, and independent, overlapping, and proxy risk factors. American Journal of Psychiatry. 2001; 158(6):848-56. [DOI:10.1176/appi.ajp.158.6.848]

[35] Frazier PA, Tix AP, Barron KE. Testing moderator and mediator effects in counseling psychology research. Journal of Counseling Psychology. 2004; 51(1):115-34. [DOI:10.1037/0022-0167.51.1.115]

[36] Spasojević J, Alloy LB. Rumination as a common mechanism relating depressive risk factors to depression. Emotion. 2001;1(1):2537. [DOI:10.1037/1528-3542.1.1.25] [PMID]

[37] Kline RB. Principles and practice of structural equation modeling. New York: Guilford; 2010.

[38] Newman MG, Zuellig AR, Kachin KE, Constantino MJ, Przeworski A, Erickson T, et al. Preliminary reliability and validity of the generalized anxiety disorder questionnaire-IV: A revised self-report diagnostic measure of generalized anxiety disorder. Behavior Therapy. 2002; 33(2):215-33. [DOI:10.1016/S00057894(02)80026-0

[39] Abdi R, Bakhshipour Roodsari A, Mahmood Alilou M, Farnam A. [Efficacy evaluation of unified transdiagnostic treatment in $\mathrm{Pa}$ tients with generalized anxiety disorder (Persian)]. International Journal of Behavioral Sciences. 2014; 11(5):245-51.

[40] Abolghasemi A, Narimani M. [Psychological tests (Persian)]. Ardebil: Bagh-e Rezvan; 2006.

[41] Bagherinejhad M, Salehi J, Tabatabayi M. [Relationship between rumination and depression in Sample of iranian students (Persian)]. Journal of Educational Psychology. 2010; 11(1):21-38.

[42] Jöreskog KG, Sörbom D. LISREL 8.80 for Windows [Computer software]. Lincolnwood: Scientific Software International, Inc; 2006.

[43] Anderson JC, Gerbing DW. Structural equation modeling in practice: A review and recommended two-step approach. Psychological Bulletin. 1988; 103(3):411-23.

[44] Chou CP, Bentler PM. Estimates and tests in structural equation modeling. In Hoyle RH, editors. Structural Equation Modeling: Concepts, Issues and Applications. Thousand Oaks, California: Sage; 1995.

[45] Kline RB. Principles and practice of structural equation modeling. New York: Guilford; 2001.

[46] Bentler PM. EQS structural equations program manual. San Fernando Valley, Los Angeles: Encino; 1995.

[47] Preacher KJ, Hayes AF. Asymptotic and resampling strategies for assessing and comparing indirect effects in multiple mediator models. Behavior Research Methods. 2008; 40(3):879-91. [PMID]

[48] Lyubomirsky S, Nolen Hoeksema S. Effects of self-focused rumination on negative thinking and interpersonal problem solving. Journal of Personality and Social Psychology. 1995; 69(1):17690. [DOI:10.1037/0022-3514.69.1.176]
[49] Davis RN, Nolen Hoeksema S. Cognitive inflexibility among ruminators and nonruminators. Cognitive Therapy and Research. 2000; 24(6):699-711.

[50] Joormann J. Differential effects of rumination and dysphoria on the inhibition of irrelevant emotional material: Ev idence from a negative priming task. Cognitive Therapy and Research. 2006; 30(2):149-60. [DOI:10.1007/s10608-006-9035-8]

[51] Moulds ML, Kandris E, Starr S, Wong ACM. The relation hip between rumination, avoidance and depression in a non-clinical sample. Behaviour Research and Therapy. 2007; 45(2):251-61. [DOI:10.1016/j.brat.2006.03.003] [PMID]

[52] Dobson KS, Dozois DJA. Risk factors in depression. New York: Elsevier; 2008.

[53] Riskind JH, Alloy LB. The Cognitive Vulnerability to Depression (CVD) Project: Current fndings and future directions. In Alloy LB, Rishkind JH, editors. Cognitive Vulnerability to Emotional Disorders. New Jersey: Paulist Press; 2006.

[54] Lyubomirsky S, Kasri F, Zehm K. Dysphoric rumination impairs concentration on academic tasks. Cognitive Therapy and Research. 2003; 27(3):309-30.

[55] Mahmood Aliloo M, Shahjoyi T, Hashemi Z. [Comparision intolerance of uncertainty, cognitive avoidance, negative attitude to problem and positive believes about worry between individua with general anxiety disorder and normal individuals (Persian)] Journal of Psychology. 2010; 5(20):20-32.

[56] Nolen-Hoeksema S. Responses to depression and their effects on the duration of depressive episodes. Journal of Abnormal Psychology. 1991; 100(4):569-582. [DOI:10.1037/0021-843X.100.4.569] [PMID]

[57] Roberts JE, Gilboa E, Gotlib IH. Ruminative response style and vulnerability to episodes of dysphoria: Gender, neuroticism, and episode duration. Cognitive Therapy and Research. 1998; 22(4):401-23.

[58] Cox BJ, Enns MW, Taylor S. The effect of rumination as a mediator of elevated anxiety sensitivity in major depression. Cognitive Therapy and Research. 2001; 25(5):525-34.

[59] Billings AG, Moos RH. Coping, stress, and social resources among adults with unipolar depression. Journal of Personality and Social Psychology. 1984; 46(4):877-91. [DOI:10.1037/00223514.46.4.877] [PMID]

[60] Nolen Hoeksema S, Wisco BE, Lyubomirsky S. Rethinking rumination. Perspectives on Psychological Science. 2008; 3(5):400 24. [DOI:10.1111/j.1745-6924.2008.00088.x] [PMID]

[61] Marroquín BM, Fontes M, Scilletta A, Miranda R. Ruminative subtypes and coping responses: Active and passive pathways to depressive symptoms. Cognition and Emotion. 2010; 24(8):144655. [DOI:10.1080/02699930903510212]

[62] Dugas MJ, RobichaudM. Cognitive-behavioral treatment for generalized anxiety disorder: From science to practice. Abingdon: Taylor \& Francis; 2007. 
This Page Intentionally Left Blank 